\title{
Magnetothermoelectric Response near Quantum Critical Points
}

\author{
M. J. Bhaseen, ${ }^{1}$ A. G. Green, ${ }^{2}$ and S. L. Sondhi ${ }^{3}$ \\ ${ }^{1}$ Theory of Condensed Matter Group, Cavendish Laboratory, Cambridge, CB3 OHE, UK. \\ ${ }^{2}$ School of Physics and Astronomy, University of St Andrews, \\ North Haugh, St Andrews, Fife, KY16 9XP, UK. \\ ${ }^{3}$ Department of Physics, Princeton University, Princeton, NJ 08544, USA.
}

(Dated: October 30, 2018)

\begin{abstract}
Following on from our previous work [Phys. Rev. Lett. 98, 166801 (2007)] we examine the finite temperature magnetothermoelectric response in the vicinity of a quantum critical point (QCP). We begin with general scaling considerations relevant to an arbitrary QCP, either with or without Lorentz invariance, and in arbitrary dimension. In view of the broad connections to high temperature superconductivity, and cold atomic gases, we focus on the quantum critical fluctuations of the relativistic Landau-Ginzburg theory. This paradigmatic model arises in many contexts, and describes the (particle-hole symmetric) superfluid-Mott insulator quantum phase transition in the Bose-Hubbard model. The application of a magnetic field opens up a wide range of physical observables, and we present a detailed overview of the charge and thermal transport and thermodynamic response. We combine several different approaches including the epsilon expansion and associated Quantum Boltzmann Equation (QBE), entropy drift, and arguments based on Lorentz invariance. The results differ markedly from the zero field case, and we include an extended discussion of the finite thermal conductivity which emerges in the presence of a magnetic field. We derive an integral equation that governs its response and explore the crossover upon changing the magnetic field. This equation may be interpreted as a projection equation in the low field limit, and clearly highlights the important rôle of collision invariants (or zero modes) in the hydrodynamic regime. Using an epsilon expansion around three-dimensions, our analytic and numerical results interpolate between our previously published value and the exact limit of two-dimensional relativistic magnetohydrodynamics.
\end{abstract}

PACS numbers: 73.43.Nq, 72.20.Pa, 74.25.Fy

\section{INTRODUCTION}

Quantum phase transitions (QPTs) in strongly correlated systems play an important rôle in modern condensed matter physics. In problems ranging from high temperature superconductivity to cold atomic gases, dramatic changes in the ground state and physical response may often accompany relatively small changes in the doping, the interaction strength, or other system parameters $\stackrel{1,2}{\underline{1}}$ With the discovery of high temperature superconductivity in the cuprates $\frac{3}{3}$ quantum phase transitions between Mott-insulators (MIs) and superfluids (SFs) or superconductors, have been at the forefront of this intense scrutiny. More recently, remarkable advances in cold atomic gases, have allowed the observation of such transitions in systems of bosonic atoms $\underline{\underline{4}}$ An important stimulus for these studies, is that the SF-MI transition separates two of the most fascinating phases of highly correlated matter. The superfluid reveals the importance of phase coherence on the macroscopic scale, and the Mott-insulator the importance of strong interactions. The transition between the two clearly involves an interplay between strong interactions and strong quantum fluctuations. Such strongly correlated regimes are notoriously difficult to analyze theoretically, and shedding light on this enigmatic transition remains challenging.

In this work, we focus on the magnetothermoelectric response in the vicinity of such quantum critical points (QCPs). Our motivation for this, and our previous work, $\frac{5}{\underline{5}}$ arose in connection with high temperature superconductivity, where experiments indicate strong superconducting fluctuations in a broad range of temperatures above $\mathrm{T}_{\mathrm{c}} \underline{\underline{6}}$ These signatures appear in both thermodynamics and transport measurements performed in magnetic fields, and include enhanced

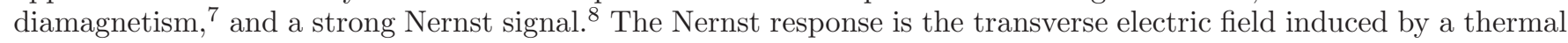
gradient in a magnetic field, and therefore hinges on the interplay of several different probes. Although a tremendous amount of theoretical progress has been made in various regions of the phase diagram, $, 10,11,12,13,14,15$ much less was known about the complete magnetothermoelectric response in the vicinity of such QCPs $\frac{16}{16}$ In view of the enhanced fluctuations, and the prospect of universal results, we advocated examining this problem at a simple, but rather generic SF-MI transition in the XY universality class $\underline{5}$ - see Fig 1 Somewhat more ambitiously, we set out with a view to describe the full complement of magnetothermoelectric response coefficients.

Our strategy ${ }^{\underline{5}}$ is to focus on the particle-hole symmetric transitions in the ubiquitous Bose-Hubbard model. This model has a distinguished history, and provides a paradigmatic example of a SF-MI quantum phase transition, $17,18,19,20,21,22,23$ While it arose primarily in connection with bosonic models of strongly correlated electron systems, where the bosons are to be thought of as Cooper pairs, it has since been cleanly realized in ground 


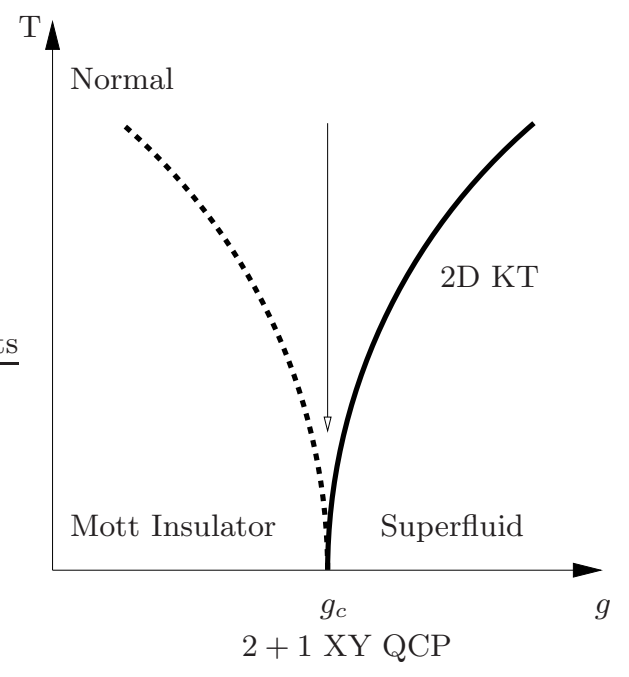

FIG. 1: Superfluid-Mott insulator quantum phase transition in $2+1$ dimensions, as tuned by a control parameter $g$, such as film thickness or doping. The diagram shows the finite temperature 2D Kosterlitz-Thouless transition, and the crossover between the Mott insulator and the normal state. We examine the universal finite temperature magnetothermoelectric response in the vicinity of the (particle-hole symmetric) $2+1$ XY QPT, as shown by the vertical arrow.

breaking experiments in cold atomic Bose gases $\underline{\underline{4}}$ Although not directly linked to a fermionic high temperature superconductor (with a d-wave order parameter and nodal quasiparticles) the simplicity of the Bose-Hubbard model is appealing. Amongst its many virtues, it admits a description as a quantum Landau-Ginzburg theory (or Abelian Higgs model) and so it naturally embraces U(1) phase fluctuations. Such fluctuations have long been argued to play an important rôle in high temperature superconductors $\stackrel{24.25}{=}$ More recently, the finite temperature classical fluctuations of the Kosterlitz-Thouless $\underline{26}$ transition have been argued to influence their diamagnetic response $\stackrel{14,15}{\underline{1}}$ In this work we focus on the vicinity of the QCP, and study the impact of quantum critical fluctuations on the fundamental transport coefficients, and other physical response functions - see Fig. 1 In contrast to other approaches, which tackle related problems from the superfluid side, and thus in terms of vortices, it is quite natural to examine the critical region in terms of particle-hole excitations of the Mott insulator. Although dual vortex formulations are possible, we do not pursue this complementary approach here.

From a condensed matter perspective, our primary interests are in the charge and thermal transport, and the thermodynamic response in the vicinity of the QCP. This critical point is of course well studied and there is a vast literature on its zero field properties which we do not attempt to review. In order to place our work in context, $\frac{5}{5}$ we simply recollect the most recent precursors pertaining to single field transport measurements in the absence of a magnetic field. It was recognized in early works that the electrical conductivity is a finite universal multiple of

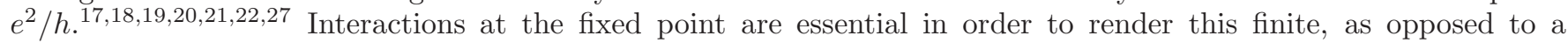
more conventional, non-interacting single carrier Drude peak. More specifically, this may be traced to collisions between counter propagating particles and holes, and in general requires a finite frequency, and finite temperature, hydrodynamic treatment of the problem $\underline{20}_{21}^{21}$ In contrast, it was well understood that the thermal conductivity diverges at the clean interacting fixed point, $; 22,28,29$ in the presence of a thermal gradient, particle and hole excitations move in the same direction, and the collision mechanism is unable to render this quantity finite. In the absence of a magnetic field, a finite thermal response therefore requires the introduction of impurities, or other forms of energy relaxation $\underline{22}$

As we noted in our previous work, $\frac{5}{-}$ this circumstance is changed markedly in the presence of a magnetic field. Although the DC Hall conductivity vanishes on symmetry grounds at the particle-hole symmetric point, the application of a magnetic field opens up the possibility of non-vanishing thermoelectric response coefficients, even in the absence of impurities, or other forms of scattering. For this reason, we confined ourselves to the clean case, in order to better expose the main universal results. This is not a serious limitation, since results in the presence of impurities may be obtained from the finite frequency behavior of the clean system, provided it's not driven to a new non-trivial fixed point. The general problem one is interested in, is thus to apply various combinations of $E, B, \nabla T$, possibly at finite frequency, and to measure the associated response coefficients. In view of the conceptual importance of the BoseHubbard model and the XY universality class, we present a variety of approaches to the general magnetothermoelectric response.

The layout of this paper is as follows: In section [I we begin with a general discussion of scaling close to a QCP. This overview is relevant to both bosonic and fermionic systems in arbitrary dimension, and thus helps to correlate 
the more detailed, model specific results we shall present. In section III we recall the field theory approach to the superfluid-insulator transition in the Bose-Hubbard model, and the simplifications at the particle-hole symmetric point. In section IV we describe the Boltzmann approach to quantum critical transport. 20.21 In sections $\mathrm{V}$, VI and VII] we present a brief overview of the properties in electric fields, magnetic fields, and temperature gradients taken separately. In section VIII we examine the behavior in combined crossed electric and magnetic fields, and discuss two distinct regimes of behavior. We approach this problem in several different ways, including entropy transport, Lorentz invariance, and a linear response analysis of the QBE. In section IX we examine the behavior in the presence of a temperature gradient and a magnetic field, and once again discuss two regimes. We verify that the Onsager relations are satisfied and obtain a non-vanishing thermal conductivity. We conclude in section XI and provide several technical appendices.

Whilst this longer manuscript was under construction, elegant extensions of this work appeared which also include the effects of impurities and particle-hole symmetry breaking at relativistic QCPs $\stackrel{30}{\underline{30}}$ These reveal important links between the transport coefficients, and develop connections to the high energy community $\underline{\underline{31}}$ Applications to other gapless systems such as graphene have also been investigated $\underline{32,33,34}$

\section{SCALING FORMS}

As usual, the approach to a continuous phase transition is accompanied by a divergent correlation length, $\xi$, and a divergent correlation time, $\xi_{\tau} \sim \xi^{z}$, where $z$ is the so-called dynamical exponent. Close to the transition, dependence on the microscopic details drops out, and non-trivial scaling relations between physical observables and the system parameters may be obtained. In the case of a quantum phase transition, where the microscopic energy scale is tuned to zero, the only relevant energy scale in the problem is the temperature, $T$. In this case, the divergent correlation time is given by

$$
\xi_{\tau} \sim 1 / T
$$

where we set, $\hbar=k_{B}=1$, for simplicity; see for example the review $\underline{\underline{2}}$ for more details. This diverging time scale is accompanied by a divergent correlation length

$$
\xi \sim\left(\xi_{t}\right)^{1 / z} \sim T^{-1 / z}
$$

where a non-trivial dynamical exponent, $z$, reflects the potential for disparity in the temporal and static correlations. Throughout this work will be interested in the magnetic, electrical and thermal response in the vicinity of a QCP. Here, we examine the general consequences which follow from simple, yet powerful, scaling ideas. As exemplified by measurements on quantum Hall systems, such considerations are able to correlate a wide variety of different physical probes, yet are crucially independent of the microscopic approach employed $\stackrel{2}{2}^{2}$

The space-time dimensions of the electric and heat currents contribute to the overall temperature dependence:

$$
\begin{aligned}
& {\left[J_{e}\right] \sim \text { Time }^{-1} \text { Length }^{-(d-1)} \sim T^{1+(d-1) / z},} \\
& {\left[J_{h}\right] \sim \text { Time }^{-2} \text { Length }^{-(d-1)} \sim T^{2+(d-1) / z} .}
\end{aligned}
$$

In general, we are also interested in the dependence of these currents on the measuring frequency and the external fields, and it is temperature against which these are compared. Having pinned the overall dimensions, these external fields

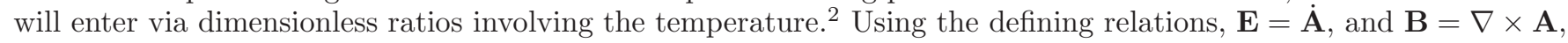

$$
\begin{aligned}
& {[\mathbf{E}] \sim \text { Length }^{-1} \text { Time }^{-1} \sim T^{1+1 / z},} \\
& {[\mathbf{B}] \sim \text { Length }^{-2} \sim T^{2 / z}}
\end{aligned}
$$

where $[\mathbf{A}]=$ Length $^{-1}$. In this way we arrive at the following scaling forms, valid in arbitrary dimension and for generic dynamical exponent:

$$
\begin{aligned}
& J_{e}(T, \mathbf{E}, \mathbf{B}, \nabla T, \omega) \sim T^{1+(d-1) / z} F_{e}\left(\frac{|\mathbf{E}|}{T^{1+1 / z}}, \frac{|\mathbf{B}|}{T^{2 / z}}, \frac{|\nabla T|}{T^{1+1 / z}}, \frac{\omega}{T}\right), \\
& J_{h}(T, \mathbf{E}, \mathbf{B}, \nabla T, \omega) \sim T^{2+(d-1) / z} F_{h}\left(\frac{|\mathbf{E}|}{T^{1+1 / z}}, \frac{|\mathbf{B}|}{T^{2 / z}}, \frac{|\nabla T|}{T^{1+1 / z}}, \frac{\omega}{T}\right),
\end{aligned}
$$


where, $\mathrm{F}_{e}$ and $\mathrm{F}_{h}$ are universality class dependent scaling functions. One may readily incorporate additional perturbations in a similar fashion. As we discuss in section [VII similar considerations also apply to thermodynamic quantities obtained from the scaling form for the free energy. We emphasize that our only assumption in deriving these scaling forms is proximity to a QCP. In particular, they are independent of the statistics of the underlying carriers, and are equally valid for both bosonic and fermionic systems. These expressions are invaluable as they enable one to confirm, and sometimes infer, the field and temperature dependence of the transport coefficients. Perhaps more importantly, they also allow one to correlate a large number of distinct scenarios and probes, without lengthy or sophisticated computations. For example, as we will discuss in section $\mathbf{\nabla}$ in the absence of an applied magnetic field, or temperature gradient, linear response in $E$ immediately yields

$$
J_{e}(T, E, \omega) \sim T^{(d-2) / z} F(\omega / T) E \equiv \sigma(\omega, T) E .
$$

The general dependence of the AC conductivity on frequency and temperature is therefore easily read off $\underline{20,21}$ Of course, in order to pin the precise functional dependence on these variables, explicit calculations of the scaling functions are necessary, and we turn our attention to this problem below. Most crucially, at low frequencies, $\omega \ll T$, collisions at the fixed point necessitate a hydrodynamic, or quantum Boltzmann treatment of the critical regime $\stackrel{20,21}{=}$ For simplicity, we consider the, $z=1$, relativistic field theory approach to the (particle-hole symmetric) SF-MI transition in the Bose-Hubbard model, but our interests, and overall approach are clearly broader.

\section{FIELD THEORY}

The Bose-Hubbard model has received considerable attention in recent years, $\underline{17,18,19,20,21,22,23}$ and describes bosons hopping on a lattice with amplitude $t$, and interacting via a short range repulsive interaction $U$ :

$$
H=-t \sum_{\langle i j\rangle}\left(b_{i}^{\dagger} b_{j}+b_{j}^{\dagger} b_{i}\right)-\mu \sum_{i} n_{i}+\frac{U}{2} \sum_{i} n_{i}\left(n_{i}-1\right) .
$$

The Bose creation and annihilation operators satisfy the usual commutation relations, $\left[b_{i}, b_{j}^{\dagger}\right]=\delta_{i j}$, where $n_{i}=b_{i}^{\dagger} b_{i}$, is the number of bosons at site $i$, and $\mu$ is the chemical potential. In the context of a Josephson array or superconductor, the bosons represent Cooper pairs of charge $Q=2 e$, tunnelling between superconducting regions. In general, one may also include the effects of disorder and long range interactions into such a model, but here we shall concentrate on the simplest case (10). The phase diagram of the Bose-Hubbard model is well established, and exhibits both superfluid and Mott insulating regions, $\frac{17}{\underline{1}}$ the latter occurring for strong enough repulsive interactions - see Fig.2. As a function of the chemical potential, $\mu$, this model exhibits a series of Mott insulating "lobes" where the density of bosons is pinned to successive integers. At a given point within the Mott lobes, the energy cost for producing particle (or hole) excitations is measured by the vertical displacement to the upper (or lower) phase boundary. At the tips of these lobes, the energy cost to producing particle-hole excitations vanishes, and the model is particle-hole symmetric. In addition, the density remains constant as one enters the superfluid phase along a trajectory of constant chemical potential, passing through this apex. In the vicinity of these points, the SF-MI transition is described by the relativistic action of an interacting complex scalar field $\Phi, \frac{17}{,}$

$$
S=\int d^{D} x\left|\partial_{\mu} \Phi\right|^{2}-m^{2}|\Phi|^{2}-\frac{u_{0}}{3}|\Phi|^{4},
$$

where $D=d+1$, and $d$ is the number of spatial dimensions of the original Bose-Hubbard model (10), and the mass parameter, $m$, is set by the temperature. This is nothing but a relativistic, quantum Landau-Ginzburg action for the superconducting order parameter, $\Phi$, and its associated fluctuations. It therefore represent a useful starting point to unravel the more general problem of phase fluctuations at SF-MI transitions. Away from these particle-hole symmetric points, the density changes as one enters the superfluid phase, and the action picks up an additional term, linear in the time derivative. Correspondingly, the dynamical exponent changes from $z=1$ to $z=2$; see for example Ref 1 . Here, we will focus on the case with $z=1$, since it will allow us to employ the powerful machinery of relativistic quantum field theory. More general results, for arbitrary $z$, may be obtained by appealing to the general scaling arguments outlined in section $\amalg$.

\section{QUANTUM BOLTZMANN EQUATION}

A convenient and physically intuitive way to think about this problem, at leading order in the epsilon expansion, is by means of the quantum Boltzmann approach to quantum critical transport $\stackrel{20,21}{=}$ In this framework we may regard 


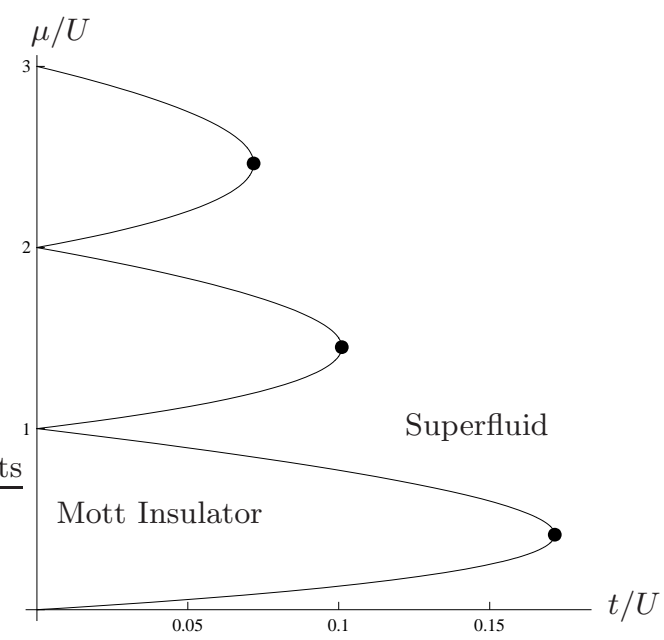

FIG. 2: Mean field phase diagram of the Bose-Hubbard model. At the tips of the Mott insulating lobes (indicated by dots) the system is particle-hole symmetric, and described by the relativistic quantum Landau-Ginzburg theory.

the model (11) as a gas of particle-hole excitations of the Mott insulator. The quantum Boltzmann equation (QBE) emerges at lowest order in the epsilon expansion and describes the impact of weak scattering (as controlled by epsilon) on these quasiparticles at the Gaussian fixed point. It takes the form of a nonlinear integro-differential equation for the momentum space distribution functions, $f_{ \pm}(\mathbf{k}, t)$, of such particle and hole excitations

$$
\frac{\partial f_{ \pm}}{\partial t} \pm Q\left(\mathbf{E}+\mathbf{v}_{\mathbf{k}} \times \mathbf{B}\right) \cdot \frac{\partial f_{ \pm}}{\partial \mathbf{k}}=\mathrm{I}_{ \pm}\left[f_{+}, f_{-}\right]
$$

where $\mathbf{v}_{\mathbf{k}} \equiv \partial \varepsilon_{\mathbf{k}} / \partial \mathbf{k}$ and $\varepsilon_{\mathbf{k}}=\sqrt{\mathbf{k}^{2} c^{2}+m^{2} c^{4}}$. For simplicity we consider a spatially homogeneous system in uniform external fields. The collision term represents scattering between these excitations, and most crucially, incorporates the nonlinear interaction of the Landau-Ginzburg field theory (11) and the associated critical fluctuations: ${ }^{20,21}$

$$
\begin{aligned}
& \mathrm{I}_{ \pm}=-\frac{2 u_{0}^{2}}{9} \int \prod_{i=1}^{3} \frac{d^{d} \mathbf{k}_{i}}{(2 \pi)^{d} 2 \varepsilon_{k_{i}}}\left(\frac{\mathcal{F}_{ \pm}^{\text {out }}-\mathcal{F}_{ \pm}^{\text {in }}}{2 \varepsilon_{k}}\right) \times \\
& (2 \pi)^{d+1} \delta\left(\mathbf{k}+\mathbf{k}_{1}-\mathbf{k}_{2}-\mathbf{k}_{3}\right) \delta\left(\varepsilon+\varepsilon_{1}-\varepsilon_{2}-\varepsilon_{3}\right),
\end{aligned}
$$

where scattering out of state $\mathbf{k}$ is given by

$$
\begin{aligned}
\mathcal{F}_{ \pm}^{\text {out }}= & 2 f_{ \pm}(\mathbf{k}) f_{\mp}\left(\mathbf{k}_{1}\right)\left[1+f_{ \pm}\left(\mathbf{k}_{2}\right)\right]\left[1+f_{\mp}\left(\mathbf{k}_{3}\right)\right] \\
& +f_{ \pm}(\mathbf{k}) f_{ \pm}\left(\mathbf{k}_{1}\right)\left[1+f_{ \pm}\left(\mathbf{k}_{2}\right)\right]\left[1+f_{ \pm}\left(\mathbf{k}_{3}\right)\right],
\end{aligned}
$$

and we have suppressed the explicit time dependence of the distribution functions. Scattering in to state $\mathbf{k}$ follows by interchanging $f_{ \pm}$and $1+f_{ \pm}$. The structure of the collision term (13) is readily seen by using Fermi's Golden rule, and represents the leading term in the $\epsilon$-expansion of the associated Keldysh field theory 21 The factors of $1+f$ remind us that we are dealing with a system of bosons in this example. As in standard treatments of $|\Phi|^{4}$ theory, it is convenient to access the non-trivial fixed point by means of an epsilon expansion around the upper critical dimension $\underline{35}$ At the Wilson-Fisher fixed point, where the renormalized mass vanishes, the bare couplings must be tuned to the values ${ }^{20}$

$$
m^{2}=\frac{4 \pi^{2} T^{2} \epsilon}{15}, \quad u_{0}=\frac{24 \pi^{2} \epsilon}{5}
$$

where our spatial dimensionality is given by $d=3-\epsilon$; since we are primarily interested in two spatial dimensions, we shall set $\epsilon=1$, at the end of any calculations. The collision term (13) is therefore proportional to $\epsilon^{2}$. As we shall see, the leading order epsilon expansion is illuminating both from a quantitative numerical perspective, and also in its ability to expose the external field and temperature dependence of physical quantities. This semiclassical approach is formally justified within the epsilon expansion where, at the temperatures of interest, the mean free path is much longer than the thermal de Broglie wavelength and the mean interparticle spacing. Further information on the relativistic QBE, $\stackrel{36}{=}$ and applications to the quark gluon plasma, may also be found in the literature $\frac{37}{\sqrt{3}}$ 
In this Boltzmann representation, the U(1) electric current of the field theory (11) takes the form

$$
\mathbf{J}_{e}=Q \int \frac{d^{d} k}{(2 \pi \hbar)^{d}} \mathbf{v}_{\mathbf{k}}\left[f_{+}(\mathbf{k}, t)-f_{-}(\mathbf{k}, t)\right]
$$

and the heat current is given (in linear response) by

$$
\mathbf{J}_{h}=\int \frac{d^{d} k}{(2 \pi \hbar)^{d}} \mathbf{v}_{\mathbf{k}} \varepsilon_{\mathbf{k}}\left[f_{+}(\mathbf{k}, t)+f_{-}(\mathbf{k}, t)\right]
$$

Here, we use $\mathbf{k}$ to label the momentum and not wavevector; the dimensions of the currents are $\left[\mathbf{J}_{e}\right]=C m^{-(d-1)} s^{-1}$ and $\left[\mathbf{J}_{h}\right]=J m^{-(d-1)} s^{-1}$. In this representation, the field theory conservation laws are related by appropriate momentum space integrals (or moments) of the QBE.

The strategy is thus the same as in any application of the Boltzmann equation. We should solve the appropriate QBE for the non-equilibrium distribution function(s), and then compute the associated transport currents. We may then extract the fundamental transport coefficients defined via

$$
\left(\begin{array}{c}
\mathbf{J}_{e}^{\mathrm{tr}} \\
\mathbf{J}_{h}^{\mathrm{tr}}
\end{array}\right)=\left(\begin{array}{cc}
\sigma & \alpha \\
\tilde{\alpha} & \bar{\kappa}
\end{array}\right)\left(\begin{array}{c}
\mathbf{E} \\
-\nabla T
\end{array}\right)
$$

where $\tilde{\alpha}=T \alpha$ is required by the Onsager relations,,$\frac{38,39,40}{4}$ and we denote the response to a temperature gradient by an overbar $\underline{\underline{60}}$ in order to distinguish it from the thermal conductivity defined in the absence of a particle current $\underline{\underline{61}}$ Knowledge of the transport coefficients is particularly useful since they allow one to correlate a wide variety of different measurements. For example, the Nernst coefficient, $\nu$, is the transverse electric field generated under open circuit conditions by a temperature gradient and a magnetic field. Imposing, $\mathbf{J}_{e}^{\mathrm{tr}}=0$, on equation (17) one may relate this open circuit measurement to the more primitive transport coefficients by $\underline{9}$

$$
\nu \equiv \frac{1}{B} \frac{E_{y}}{(-\nabla T)_{x}}=\frac{1}{B} \frac{\alpha_{x y} \sigma_{x x}-\alpha_{x x} \sigma_{x y}}{\sigma_{x x}^{2}+\sigma_{x y}^{2}} .
$$

At the particle-hole symmetric point we have chosen to focus on, the Hall coefficient, $\sigma_{x y}$, vanishes and this may be reduced to

$$
\nu=\frac{1}{B} \frac{\alpha_{x y}}{\sigma_{x x}} .
$$

Such an approximate reduction may also occur in situations where $\sigma_{x y} \approx 0$. We see that the transverse thermoelectric response, $\alpha_{x y}$, is central to a discussion of the Nernst response. We shall examine this quantity in detail from several different perspectives, beginning in section VIII We shall also discuss the behavior of the other transport coefficients, including the thermal conductivity, $\bar{\kappa}_{x x}(B)$.

\section{ELECTRIC FIELD RESPONSE}

Before embarking on a detailed discussion of the general magnetothermoelectric response, it is instructive to recall the main known results pertaining to single field measurements $\frac{17,18,20}{1,2}$ As we discussed in section [I] in the absence of any applied temperature gradients or magnetic fields, one expects the linear response electric current to behave as

$$
J_{e}(T, \mathbf{E}, \omega) \sim T^{1+(d-1) / z} \frac{|\mathbf{E}|}{T^{1+1 / z}} \Sigma\left(\frac{\omega}{T}\right) .
$$

The corresponding conductivity therefore reads

$$
\sigma(T, \omega) \sim T^{(d-2) / z} \Sigma\left(\frac{\omega}{T}\right) .
$$

For $z=1$, this is borne out by the direct Boltzmann calculations of Damle and Sachdev $\stackrel{20}{n}$ Indeed, the nontrivial scaling (21) as a function of $\omega / T$ was an important catalyst for their Boltzmann treatment; in general the limits $\omega \rightarrow 0$ and $T \rightarrow 0$ do not commute and $\Sigma(0) \neq \Sigma(\infty)$. As such the DC response at finite temperature differs from that obtained at zero temperature. The universal scaling function, $\Sigma(\omega / T)$, may be computed numerically in the low frequency hydrodynamic regime by means of the QBE (12), and at zero frequency one obtains $\underline{20,21}$

$$
\Sigma(0)=\frac{0.1650}{\epsilon^{2}} .
$$


In $d=2$, where $\epsilon=1$, the DC conductivity is a universal multiple of $e^{2} / h$ :

$$
\sigma(0)=\Sigma(0) \frac{(2 e)^{2}}{\hbar} \simeq 1.037 \frac{4 e^{2}}{h} .
$$

This result is clearly appealing, and is remarkably close to both the self-dual value $\stackrel{42,43}{\underline{4}} 4 e^{2} / h$, and to a number of early experiments on thin films ${ }^{20}$ More generally, the order of limits is also relevant to the electric field itself. A discussion of the non-commutativity of $E, \omega, T$, and the associated non-linear response, may be found in Refs. 22 23.

It is worth noting that the result (22) is singular in the small parameter $\epsilon$. This is a direct reflection of the proximity to the Gaussian fixed point, and that collisions must overcome the conventional Drude response for a single non-interacting carrier. This is possible in this two carrier system because electric current relaxation does not violate momentum conservation ${ }^{20}$ A steady state with a finite conductivity is therefore established. As we shall see, the specific leading order dependence on $\epsilon$ is intimately tied to the physical quantity under consideration. For example, in section VIII we shall demonstrate that the leading order contribution to $\alpha_{x y}$ is regular in the epsilon expansion. To leading order, one may therefore drop the $\mathcal{O}\left(\epsilon^{2}\right)$ collision term in the QBE and consider bosons of mass $\mathcal{O}(\sqrt{\epsilon})$ moving under the action of the applied fields. This is in stark contrast to the case considered by Damle and Sachdev $\stackrel{20}{=}$ where the collision term was crucial in order to render the electrical conductivity finite and proportional to $1 / \epsilon^{2}$. Nonetheless, as we shall discuss below, the collision term is central to a better understanding of the thermal conductivity in the drift regime. Here the leading order contribution begins at $\mathcal{O}\left(\epsilon^{2}\right)$ and is inversely related to the electrical conductivity. For uniformity of presentation we shall include the collision term throughout, and simplify when it is appropriate.

\section{TEMPERATURE GRADIENT RESPONSE}

In view of the finite DC electrical conductivity discussed above, it is tempting to think that the system might also support a finite thermal conductivity, in the spirit of a conventional Wiedemann-Franz relation. A little reflection reveals that this is not the case however, and that the thermal conductivity in fact diverges in the clean system. ${ }^{28}$ A simple way to see this is to note that under the action of the thermal gradient, particles and holes move in the same direction. As such, relaxation of the corresponding heat current requires energy relaxation, which is not contained in either the original model (11) or the Boltzmann description (12). (This is in contrast to the electrical conductivity, where particles and holes move in opposite directions, with no net momentum. Collisions are highly effective in limiting the electrical current, without violating the conservation laws $\stackrel{20}{2}$ ) It follows that the divergence of the thermal conductivity is intimately tied to the conservation of the energy momentum tensor, 28 in much the same

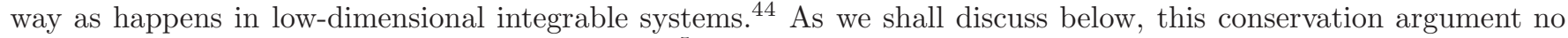
longer applies in the presence of a magnetic field $\underline{\underline{5}}^{\mathrm{b}}$ By minimally coupling the field theory (11) to a magnetic field, the conservation law is modified. As such, in the presence of a magnetic field, a non-vanishing thermoelectric tensor and thermal conductivity may be supported, even in the clean homogeneous system $\underline{\underline{5}}$ Impurities and other scattering mechanisms such as umklapp processes, may of course render these quantities finite also, but in the first instance, it is clearly essential to understand the universal results pertaining to the clean homogeneous system. Before embarking on a general discussion of the interplay between a magnetic field and other probes and response functions, let us examine the response to a magnetic field alone.

\section{MAGNETIC FIELD RESPONSE}

In view of the interest in fluctuation diamagnetism $\frac{14,15}{10}$ it is instructive to investigate the response to a magnetic field alone. Recalling our scaling arguments from section [II in the vicinity of a QCP, the only relevant energy scale against which to compare the magnetic field is the temperature. In the absence of electrical and thermal gradients, we therefore expect the free energy density to scale as ${ }^{5}$

$$
\mathcal{F}(T, B) \sim T^{1+d / z} f_{1}\left(\frac{B}{T^{2 / z}}\right) .
$$

Here we have used the fact that the correlation length, $\xi$, diverges with the correlation time, $\xi_{\tau} \sim 1 / T$, according to $\xi \sim\left(\xi_{\tau}\right)^{1 / z}$, and so the prefactor is an energy density. In addition, we have used the fact that $[B] \sim 1 / L^{2} \sim T^{2 / z}$, as follows from our discussion in section [II In weak magnetic fields, $B \ll T^{2 / z}$, we may expand this in powers of $B$ :

$$
\mathcal{F}(T, B) \sim T^{1+(d-4) / z} B^{2} .
$$


Here we assume that symmetry under reversal of the magnetic field ensures that only even powers of $B$ appear in the weak field expansion of the scaling function $f_{1}$. It follows that the linear response magnetization scales as ${ }^{5}$

$$
M=-\frac{\partial \mathcal{F}}{\partial B} \sim T^{1+(d-4) / z} B .
$$

This is consistent with a finite temperature, diagrammatic Kubo calculation of the magnetic susceptibility of a charged scalar field, with $m \sim T$ and $z=1$. On the other hand, in strong magnetic fields, $B \gg T^{2 / z}$, it is natural to expand this in a power series in temperature, and recast the scaling relation (24) in the form

$$
\mathcal{F}(T, B) \sim B^{(d+z) / 2} \tilde{f}_{1}\left(\frac{T}{B^{z / 2}}\right) .
$$

In strong fields, or low temperatures, $B \gg T^{2 / z}$, we thus expect

$$
\mathcal{F}(T, B) \sim B^{(d+z) / 2}
$$

In the relativistic problem, this yields the strong field behavior, $\mathcal{F} \sim B^{3 / 2}$, in $d=2$, and $\mathcal{F} \sim B^{2}$, in $d=3$. Broadly speaking, this non-trivial field dependence is a reflection of the relativistic Landau level spectrum, where $\mathcal{E} \sim \sqrt{B}$. This is suggested on dimensional grounds by equation (24) for $z=1$, and is borne out in a direct computation of the partition function of a free massive relativistic charged scalar field. $\stackrel{45,46}{ }$ These considerations are also compatible with elegant and highly non-trivial results obtained in the early days of quantum electrodynamics on vacuum polarization and pair production; both spinor and scalar quantum electrodynamics 62 These detailed studies require ultraviolet regularization and yield an additional logarithm at strong magnetic fields, so that $\mathcal{F} \sim B^{2} \ln (1 / B)$ in $d=3$. Although this is not captured by the simple scaling arguments, it is intimately connected to the vacuum screening properties of the field theory via the renormalization group beta function.

In this section we have discussed the distinct field regimes of the static magnetic response. In the subsequent discussion, we shall see how such regime divisions also emerge in the transport properties. In many respects this is rather natural, since the dynamics and statics are intimately related at QCPs $\stackrel{2}{2}$

\section{CROSSED ELECTRIC AND MAGNETIC FIELDS}

In order to understand the quantum critical transport in combined $\mathbf{E}$ and $\mathbf{B}$ fields, it is instructive to recall the motion of a single relativistic particle in crossed electric and magnetic fields. 51 This will provide significant orientation for the more general interacting field theory,$\underline{\underline{5}}$ The most important feature is that the motion of a single charged particle is qualitatively different depending on whether, $E<c_{0} B$, or $E>c_{0} B$, where $c_{0}$ is the speed of light. This is most easily understood from the vantage point of a moving frame of reference 51 In the former case, there always exists a moving frame where the electric field vanishes, and the particle experiences a pure magnetic field. Conversely, in the latter case, there always exists a frame where the magnetic field vanishes, and the particle experiences a pure electric field. Boosting back to the lab frame, we mix in the complementary field component, but the qualitative character of the motion is more "electric field like" or "magnetic field like", as dictated by the inequalities — see Fig. 3 . We shall discuss this in more detail below, but before doing so, we note that our problem is a little more subtle due to the appearance of an effective speed of light, $c$, in the effective field theory (11), and the associated QBE (12). What matters for our purposes, at least in the first instance, is the solutions to the QBE (12) for a given fixed ratio of $E$ and $B$. It may be seen that these solutions are kinematically distinct for, $E<c B$, and $E>c B$, as follows directly from the left hand side of the differential equation, without recourse to Lorentz invariance arguments. As, such it the effective Lorentz structure which plays a key rôle in determining the solutions of the relativistic QBE (12), and we shall henceforth use this effective speed of light in our subsequent discussions. It is interesting to note that such Lorentz transformation arguments also find applications in other effective relativistic systems, as was recently discussed by Lukose et al in the context of graphene $\underline{\underline{52}}$

Under a Lorentz transformation with a velocity $\mathbf{v}$, the electric and magnetic fields transform according to 51

$$
\begin{aligned}
& \mathbf{E}^{\prime}=\gamma(\mathbf{E}+\mathbf{v} \times \mathbf{B})-\frac{\gamma^{2}}{\gamma+1} \frac{\mathbf{v}(\mathbf{v} \cdot \mathbf{E})}{c^{2}}, \\
& \mathbf{B}^{\prime}=\gamma\left(\mathbf{B}-\frac{\mathbf{v} \times \mathbf{E}}{c^{2}}\right)-\frac{\gamma^{2}}{\gamma+1} \frac{\mathbf{v}(\mathbf{v} \cdot \mathbf{B})}{c^{2}},
\end{aligned}
$$


where, $\gamma=1 / \sqrt{1-v^{2} / c^{2}}$, and we work in SI units. It is readily seen that for crossed $\mathbf{E}$ and $\mathbf{B}$ fields, with $|\mathbf{E}|<c|\mathbf{B}|$, there exists a frame moving at the drift velocity

$$
\mathbf{v}_{\mathrm{D}} \equiv \frac{\mathbf{E} \times \mathbf{B}}{|\mathbf{B}|^{2}}
$$

where the electric field $\mathbf{E}^{\prime}$ vanishes. In this moving frame, the particle is subject to a pure magnetic field of reduced strength, $\mathbf{B}^{\prime}=\mathbf{B} / \gamma_{\mathrm{D}}$. Taking our magnetic field to point along the z-axis, the particle executes cyclotron orbits in the moving frame with, $x^{\prime}\left(t^{\prime}\right)=r \cos \left(\omega^{\prime} t^{\prime}\right)$ and $y^{\prime}\left(t^{\prime}\right)=\mp r \sin \left(\omega^{\prime} t^{\prime}\right)$; here $r$ is the radius of the orbit, $\omega^{\prime}$ is the cyclotron frequency, and the signs indicate the sense of rotation for positive and negative charges respectively. With the electric field along the $x$-axis, the drift velocity points along the negative $y$-axis. Boosting back to the lab frame using the inverse Lorentz transformations one finds the parametric equations of motion:

$$
x(t)=r \cos \left(\omega^{\prime} t^{\prime}\right), \quad y(t)=\gamma\left(\mp r \sin \left(\omega^{\prime} t^{\prime}\right)-v_{D} t^{\prime}\right), \quad t=\gamma\left(t^{\prime} \pm v_{\mathrm{D}} r \sin \left(\omega^{\prime} t^{\prime}\right) / c^{2}\right) .
$$

In the nonrelativistic limit $c \rightarrow \infty$, these reduce to the parametric equations of a trochoid: $x^{2}(t)+\left(y(t)+v_{\mathrm{D}} t\right)^{2}=r^{2}-$ see Fig 3. In view of the cyclotron motion of equal numbers of particles and holes, it follows that the DC conductivity, $\sigma_{x x}(B)=0$, in this regime, at least at the single particle level. On the other hand we see that the thermoelectric tensor, $\alpha_{x y}$, may be finite due to the finiteness of the drift velocity. We shall see that these expectations are borne out, even in the presence of interactions at the fixed point, and that this single particle description captures the relevant physics $\stackrel{\underline{5}}{\underline{5}}$ It also underpins the divergence of the Nernst coefficient (19) in the clean, particle-hole symmetric case $\underline{\underline{5}}$

Returning to our single particle problem, for crossed $\mathbf{E}$ and $\mathbf{B}$ fields with $|\mathbf{E}|>c|\mathbf{B}|$, there exists a frame moving at velocity

$$
\mathbf{v}_{\mathrm{B}} \equiv c^{2}\left(\frac{\mathbf{E} \times \mathbf{B}}{|\mathbf{E}|^{2}}\right),
$$

where the magnetic field $\mathbf{B}^{\prime}$ vanishes. In this moving frame, the charged particle is subject to a pure electric field of reduced strength, $\mathbf{E}^{\prime}=\mathbf{E} / \gamma_{\mathrm{B}}$. In the absence of scattering, the energy $\varepsilon^{\prime}$, and the components of momentum $\mathbf{p}^{\prime}$ parallel to $\mathbf{E}$, continue to increase indefinitely. In the lab frame

$$
\begin{aligned}
\varepsilon & =\gamma_{\mathrm{B}}\left(\varepsilon^{\prime}+\mathbf{v}_{\mathrm{B}} \cdot \mathbf{p}^{\prime}\right), \\
\mathbf{p}_{\|} & =\gamma_{\mathrm{B}}\left(\mathbf{p}_{\|}^{\prime}+\mathbf{v}_{\mathrm{B}} \varepsilon^{\prime} / c^{2}\right), \\
\mathbf{p}_{\perp} & =\mathbf{p}_{\perp}^{\prime},
\end{aligned}
$$

where the labels parallel and perpendicular are with respect to the boost velocity, $\mathbf{v}_{\mathrm{B}}$. In these notations $\mathbf{p}_{\|}^{\prime}$ is constant (since it is transverse to the electric field) whilst $\mathbf{p}_{\perp}^{\prime}$ and $\varepsilon^{\prime} \equiv \sqrt{c^{2} \mathbf{p}^{2}+m^{2} c^{4}}$ increase with time. It follows from equations (34), (35) and (36) that the energy, and both components of the momentum increase indefinitely in the lab frame. That is to say, if we apply an electric field $|\mathbf{E}|>c|\mathbf{B}|$, both particles and holes acquire an identical and ever increasing component of the momentum at right angles to the electric field ${ }^{63}$ This cannot be relaxed by the collision term. Since, $\varepsilon_{k} \mathbf{v}_{k}=c^{2} \mathbf{k}$, we expect that $\alpha_{x y}$ is infinite in this regime. On the other hand, compatibility with the results of Damle and Sachdev, for $\mathbf{B}=0$, suggests the possibility of a finite value of $\sigma_{x x}$. Once again, the underlying divergence of the Nernst coefficient (19) is apparent.

From the preceeding discussion, we see how the ratio, $E / B$, may influence transport measurements. As we shall discuss in section IX, there are analogous regimes in a thermal gradient, for $\nabla T \lesssim B$, and $\nabla T \gtrsim B$, at least within the framework of the collisionless Boltzmann equation with a linearized driving term. Although we no longer have Lorentz invariance arguments, the distinction once again shows up in the single particle kinematics of the associated $\mathrm{QBE}$, since the thermal gradient acts like a momentum-dependent electric field. In the remainder of this section, we shall take the electric field regimes in turn, and examine the magnetothermoelectric transport coefficients from a variety of different perspectives. We will focus primarily on the drift regime, $|\mathbf{E}|<c|\mathbf{B}|$, since it is both the simplest to analyze, and also pertains to conventional linear response measurements at fixed magnetic field.

\section{A. Drift Regime: $|\mathbf{E}|<c|\mathbf{B}|$}

In this section we are interested in the thermoelectric response in the drift regime. It turns out that there are several complementary ways to address this problem, and we examine these below. Our strategy is to first develop an understanding of the QBE (12) as written, and to defer discussion of the epsilon expansion itself until later. This 

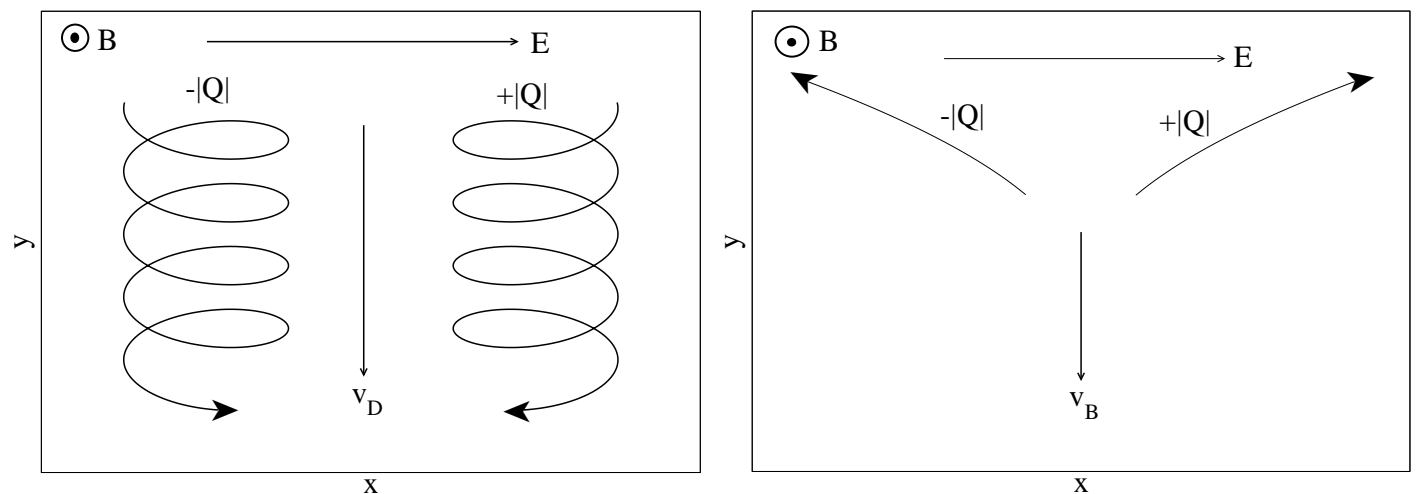

FIG. 3: Motion of a single relativistic charged particle in crossed electric and magnetic fields. (a) In the regime $|\mathbf{E}|<c|\mathbf{B}|$ the particle executes cyclotron orbits and has a well defined transverse drift velocity $v_{D} \cdot[(\mathrm{b})]$ In the regime $|\mathbf{E}|>c|\mathbf{B}|$ the particle is continually accelerated by the electric field.

combination of different perspectives is particularly useful in establishing the Onsager relations between the transport coefficients $\frac{38,39,40}{1 t}$ It also helps demonstrate the equivalence between the field theory and quantum Boltzmann approaches. We begin in section VIII A 1 with a computation of the thermoelectric tensor, $\alpha_{x y}$, based on the entropy drift of a charged scalar field. In section VIII A 2 we turn our attention to the more general problem of the Lorentz invariant solutions of the QBE. In section VIII A3 we use this explicit distribution to determine the heat current response to an electric field, and verify the answer obtained by entropy drift. In sectionVIII A4 we demonstrate how this result also follows from a more familiar linearization of the QBE. Such a linearization approach will be particularly useful in the presence of thermal gradients, where Lorentz field transformation arguments are not available. In section VIIIA 5 we finally turn to the epsilon expansion itself, and evaluate our general expression for $\alpha_{x y}$ numerically. We compare the results to our scaling analysis of section [I] We provide a very brief discussion of the non-drift regime in section VIIIB

\section{Entropy Flow}

As follows from the defining relations (17), the thermoelectric tensor, $\alpha$, quantifies the electric current which flows in response to a temperature gradient. Provided the Onsager relations are satisfied, this may also be obtained (up to a factor of temperature) from the heat current which flows in response to a temperature gradient. The latter route is easier to begin with, and we consider the complementary approach in section IX In view of the well defined drift velocity it is natural to compute the transverse thermoelectric response, $\alpha_{x y}$, as a transport of entropy with the characteristic flow

$$
\mathbf{v}_{\mathrm{D}}=\frac{\mathbf{E} \times \mathbf{B}}{|\mathbf{B}|^{2}}
$$

To lowest order in the epsilon expansion, it is sufficient to compute the entropy density of a free massive charged scalar field, where the mass parameter is given by (14). That is to say, the mass incorporates the leading Hartree contribution of the self-interactions $\frac{20}{2} \mathrm{~A}$ free boson is essentially a harmonic oscillator, and the partition function of the latter is readily seen to be

$$
Z=\sum_{n=0}^{\infty} e^{-\beta(n+1 / 2) \hbar \omega}=\frac{1}{2 \sinh \left(\frac{\beta \hbar \omega}{2}\right)} .
$$

Taking the logarithm and integrating over all momenta, the analogous expression for a massive neutral scalar field follows immediately: $\stackrel{53}{=}$

$$
\ln Z=-V \int \frac{d^{d} k}{(2 \pi \hbar)^{d}}\left(\frac{\beta \hbar \omega_{k}}{2}+\ln \left(1-e^{-\beta \hbar \omega_{k}}\right)\right)
$$

where $\hbar \omega_{k} \equiv \varepsilon_{k}=\sqrt{k^{2} c^{2}+m^{2} c^{4}}$. Dropping the first (divergent zero point) contribution, the free energy density reads

$$
\mathcal{F}=k_{B} T \int \frac{d^{d} k}{(2 \pi \hbar)^{d}} \ln \left(1-e^{-\beta \varepsilon_{k}}\right) .
$$


The corresponding entropy density is given by

$$
\mathcal{S}=-\frac{\partial \mathcal{F}}{\partial T}=-k_{B} \int \frac{d^{d} k}{(2 \pi \hbar)^{d}}\left[\ln \left(1-e^{-\beta \varepsilon_{k}}\right)-\frac{\beta \varepsilon_{k}}{e^{\beta \varepsilon_{k}}-1}\right] .
$$

Performing an integration by parts on the logarithmic term one obtains

$$
\mathcal{S}=\frac{1}{d T} \int \frac{d^{d} k}{(2 \pi \hbar)^{d}} f_{0}\left(\varepsilon_{k}\right) \nabla_{\mathbf{k}} \cdot\left(\varepsilon_{k} \mathbf{k}\right)
$$

where $f_{0}=\left(e^{\beta \varepsilon_{k}}-1\right)^{-1}$ is the Bose distribution function, and we have used the identity $\nabla_{\mathbf{k}} \cdot\left(\varepsilon_{k} \mathbf{k}\right)=\mathbf{v}_{\mathbf{k}} \cdot \mathbf{k}+d \varepsilon_{k}$. Since particles and holes acquire the same drift velocity, the corresponding heat current density for our charged scalar field theory is given by $\mathbf{J}_{h}=2 T \mathcal{S}_{\mathbf{v}_{\mathrm{D}}}$. In particular, if we apply an electric field, $E_{x}$, in the positive $x$-direction, and a magnetic field, $B_{z} \equiv B$, in the positive $z$-direction, the drift velocity points in the negative $y$-direction:

$$
J_{h}^{y}=-2 T \mathcal{S} \frac{E_{x}}{B_{z}}
$$

Assuming the validity of the Onsager relations, which we demonstrate are satisfied in section IX. we thus obtain

$$
\alpha_{y x}=-\frac{2 \mathcal{S}}{B}
$$

Equivalently, using the Onsager symmetry relation ${ }^{38} \alpha_{x y}(\mathbf{B})=\alpha_{y x}(-\mathbf{B})$

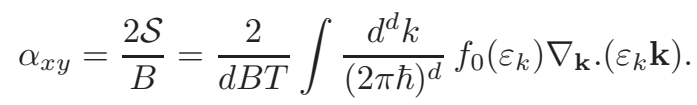

A useful alternative form of this result, which better exposes the relation to heat currents, is obtained by performing another integration by parts:

$$
\alpha_{x y}=\frac{2}{d B T} \int \frac{d^{d} k}{(2 \pi \hbar)^{d}} \varepsilon_{k} \mathbf{k} \cdot\left(-\nabla_{\mathbf{k}} f_{0}\right)
$$

Using the identity

$$
\nabla_{\mathbf{k}} f_{0}=\frac{c^{2} \mathbf{k}}{\varepsilon_{k}}\left(\frac{\partial f_{0}}{\partial \varepsilon_{k}}\right)
$$

we may also write this in scalar form as

$$
\alpha_{x y}=\frac{2 \mathcal{S}}{B}=\frac{2 c^{2}}{d B T} \int \frac{d^{d} k}{(2 \pi \hbar)^{d}} k^{2}\left(-\frac{\partial f_{0}}{\partial \varepsilon_{k}}\right) .
$$

Note that the result for $\alpha_{x y}$ is positive in sign; an electric field in the $y$-direction and a magnetic field in $z$-direction yields $\mathbf{E} \times \mathbf{B}$ drift, and thus a transport of heat, in the positive $x$-direction. In the next sections we shall see how this result (48) emerges from the QBE (12), even in the presence of interactions. Developing the Boltzmann approach is particularly useful since it will allow an intuitive and systematic calculation of all the transport coefficients in this regime. Before closing the section let us note that the entropy density per species of carrier has a particularly simple form in the massless limit. With $m=0$ one obtains

$$
\mathcal{S}=\mathcal{C}_{d}\left(k_{B} \lambda_{T}^{-d}\right)
$$

where $\lambda_{T} \equiv \hbar c / k_{B} T$ is a thermal wavelength for massless particles, and

$$
\mathcal{C}_{d} \equiv \frac{1}{d} \int \frac{d^{d} \bar{k}}{(2 \pi)^{d}} \frac{\bar{k}^{2} e^{\bar{k}}}{\left(e^{\bar{k}}-1\right)^{2}}
$$

where $\bar{k} \equiv c k / k_{B} T$ are dimensionless variables. In particular, $\mathcal{C}_{3}=2 \pi^{2} / 45 \approx 0.439$, and $\mathcal{C}_{2}=3 \zeta(3) / 2 \pi \approx 0.574$. We shall employ the first of these results within the epsilon expansion in section VIII A 5. 


\section{Lorentz Invariance and the $Q B E$}

The first step in developing the Boltzmann approach is to solve the QBE for the non-equilibrium distribution functions, $f_{ \pm}(\mathbf{k}, t)$. In the absence of any electric and magnetic fields, it is readily verified that the QBE (12), is satisfied by the static Bose distribution function

$$
f_{ \pm}(\mathbf{k}, t)=f_{0}\left(\varepsilon_{\mathbf{k}}\right) \equiv \frac{1}{e^{\beta \varepsilon_{\mathbf{k}}}-1},
$$

even in the presence of the collision term. This is easily seen by noting that $1+f_{0}\left(\varepsilon_{\mathbf{k}}\right)=e^{\beta \varepsilon_{\mathbf{k}}} f_{0}\left(\varepsilon_{\mathbf{k}}\right)$. It follows that $\mathcal{F}_{ \pm}^{\text {out }}$ equals $\mathcal{F}_{ \pm}^{\text {in }}$ provided $\varepsilon(\mathbf{k})+\varepsilon\left(\mathbf{k}_{1}\right)=\varepsilon\left(\mathbf{k}_{2}\right)+\varepsilon\left(\mathbf{k}_{3}\right)$. The energy conserving delta function present in equation (13) is therefore enough to ensure that the collision term vanishes and that the full QBE is satisfied.

Let us now examine the non-equilibrium situation in the presence of crossed electric and magnetic fields. As discussed in section VIII, in the regime $|\mathbf{E}|<c|\mathbf{B}|$, we may move to a frame with velocity

$$
\mathbf{v}_{\mathrm{D}}=\frac{\mathbf{E} \times \mathbf{B}}{|\mathbf{B}|^{2}},
$$

where the electric field vanishes. Since a pure magnetic field does not affect the energy of a charged particle, it follows than an equilibrium distribution $f_{0}\left(\varepsilon_{\mathbf{k}}^{\prime}\right)$ must satisfy the Boltzmann equation in this boosted frame. (Indeed, it is readily verified by direct substitution that $f_{ \pm}(\mathbf{k})=f_{0}\left(\varepsilon_{\mathbf{k}}\right)$ is a solution of the original QBE (12) when $\mathbf{E}=0$ and $\mathbf{B} \neq 0$, since $\partial f_{0} / \partial \mathbf{k}=\mathbf{v}_{k} \partial f_{0} / \partial \varepsilon_{\mathbf{k}}$.) Since

$$
\varepsilon_{\mathbf{k}}^{\prime}=\gamma_{\mathrm{D}}\left(\varepsilon_{\mathbf{k}}-\mathbf{v}_{\mathrm{D}} \cdot \mathbf{k}\right)
$$

where, $\gamma_{\mathrm{D}}=\left(1-\mathbf{v}_{\mathrm{D}}^{2} / c^{2}\right)^{-1 / 2}$, we conclude that

$$
f_{ \pm}(\mathbf{k})=f_{0}\left(\varepsilon_{\mathbf{k}}^{\prime}\right)=f_{0}\left(\frac{\varepsilon_{\mathbf{k}}-\mathbf{v}_{\mathrm{D}} \cdot \mathbf{k}}{\sqrt{1-\mathbf{v}_{\mathrm{D}}^{2} / c^{2}}}\right),
$$

is a solution of the full QBE (12). Again, it may be verified by direct substitution that (54) is in fact a solution of (12) in the presence of our crossed $\mathbf{E}$ and $\mathbf{B}$ fields. Explicitly, the left hand side of the Boltzmann equation is readily seen to give zero using the fact that $\mathbf{B} \times \mathbf{v}_{\mathbf{D}}=\mathbf{E}$ :

$$
\left(\mathbf{E}+\mathbf{v}_{\mathbf{k}} \times \mathbf{B}\right) \cdot \frac{\partial f_{ \pm}}{\partial \mathbf{k}}=\left(\mathbf{E}+\mathbf{v}_{\mathbf{k}} \times \mathbf{B}\right) \cdot\left(\mathbf{v}_{\mathbf{k}}-\mathbf{v}_{\mathrm{D}}\right) \gamma_{\mathrm{D}} \frac{\partial f_{0}\left(\varepsilon_{\mathbf{k}}^{\prime}\right)}{\partial \varepsilon_{\mathbf{k}}^{\prime}} \propto \mathbf{v}_{\mathbf{k}} \cdot\left(\mathbf{E}-\mathbf{B} \times \mathbf{v}_{\mathrm{D}}\right)=0 .
$$

Likewise, in the collision term we see that $\mathcal{F}_{ \pm}^{\text {out }}$ equals $\mathcal{F}_{ \pm}^{\text {in }}$ provided that $\varepsilon^{\prime}(\mathbf{k})+\varepsilon^{\prime}\left(\mathbf{k}_{1}\right)=\varepsilon^{\prime}\left(\mathbf{k}_{2}\right)+\varepsilon^{\prime}\left(\mathbf{k}_{3}\right)$. Since $\varepsilon^{\prime}(\mathbf{k})=\gamma_{\mathrm{D}}\left(\varepsilon_{\mathbf{k}}-\mathbf{v}_{\mathrm{D}} \cdot \mathbf{k}\right)$, this is ensured by the combined action of the energy and momentum conserving delta functions appearing in (13). In this way we have established the non equilibrium solution (54) of the QBE valid in the drift regime $|\mathbf{E}|<c|\mathbf{B}|$. This is analogous to Kohn's theorem ${ }^{54}$ for non-relativistic electron systems $\underline{55}^{5 n}$ the next section we shall use this distribution to compute the transport coefficients.

\section{Transverse heat current in response to an electric field}

The equality of the particle and hole distribution functions (54) reflects the fact that drift velocity (52) is independent of the charge of the carrier. It follows immediately from the definition (15), that the both the longitudinal and the transverse components of the electrical conductivity vanish in this limit. Note that there is no conflict with the existing results of Damle and Sachdev, 20,21 since our present results are derived in the drift regime $|\mathbf{E}|<c|\mathbf{B}|$. As such we cannot simply set $\mathbf{B}=0$ and recover the results obtained in the absence of $\mathbf{B}$. Moreover, the vanishing of the conductivities are consistent with the single particle picture presented in Fig. 3, In contrast, it is clear from the definition (16), that a non-vanishing heat current may be supported in crossed $\mathbf{E}$ and $\mathbf{B}$ fields. Substituting the drift solution (54) into (16) yields

$$
\mathbf{J}_{h}=2 \int \frac{d^{d} k}{(2 \pi \hbar)^{d}} c^{2} \mathbf{k} f_{0}\left(\frac{\varepsilon_{\mathbf{k}}-\mathbf{v}_{\mathrm{D}} \cdot \mathbf{k}}{\sqrt{1-\mathbf{v}_{\mathrm{D}}^{2} / c^{2}}}\right)
$$


Taylor expanding the distribution function in powers of $\mathbf{v}_{\mathrm{D}}$ gives

$$
\mathbf{J}_{h}=2 \int \frac{d^{d} k}{(2 \pi \hbar)^{d}} c^{2} \mathbf{k}\left[f_{0}\left(\varepsilon_{\mathbf{k}}\right)+\frac{\beta e^{\beta \varepsilon_{\mathbf{k}}}}{\left(e^{\beta \varepsilon_{\mathbf{k}}}-1\right)^{2}} \mathbf{k} \cdot \mathbf{v}_{\mathrm{D}}+\mathcal{O}\left(\mathbf{v}_{\mathrm{D}}^{2}\right)\right]
$$

where the first term vanishes upon integration. In the presence of an electric field, $E_{x}$, and a magnetic field, $B_{z} \equiv B$, the drift velocity $\mathbf{v}_{\mathrm{D}}$ is in the negative y-direction. As such, to linear order in the electric field

$$
J_{h}^{y}=-2 \int \frac{d^{d} k}{(2 \pi \hbar)^{d}} c^{2} k_{y}^{2} \frac{\beta e^{\beta \varepsilon_{\mathbf{k}}}}{\left(e^{\beta \varepsilon_{\mathbf{k}}}-1\right)^{2}} \frac{E_{x}}{B_{z}} .
$$

It follows from the defining relations (17) that

$$
\tilde{\alpha}_{y x}=-\frac{2}{d} \int \frac{d^{d} k}{(2 \pi \hbar)^{d}} c^{2} k^{2} \frac{\beta e^{\beta \varepsilon_{\mathbf{k}}}}{\left(e^{\beta \varepsilon_{\mathbf{k}}}-1\right)^{2}} \frac{1}{B_{z}} .
$$

Equivalently, using the Onsager relations ${ }^{38} \tilde{\alpha}_{y x}(\mathbf{B})=T \alpha_{y x}(\mathbf{B})$ and $\alpha_{x y}(\mathbf{B})=\alpha_{y x}(-\mathbf{B})$

$$
\alpha_{x y}=\frac{2 c^{2}}{d B T} \int \frac{d^{d} k}{(2 \pi \hbar)^{d}} k^{2}\left(-\frac{\partial f_{0}}{\partial \varepsilon_{\mathbf{k}}}\right) .
$$

This coincides with our previous result (48) obtained by entropy drift arguments. This supports the validity of our simple result, that at the strongly fluctuating SF-MI transition, $\alpha_{x y}=2 \mathcal{S} / B$, where $\mathcal{S}$ is the total entropy density pertaining to each carrier type.$^{5}$

\section{Linear Response}

In order to go beyond our existing results, especially with a view to thermal gradients in section IX. it is useful to perform a systematic linear analysis of the QBE. In the presence of electric and magnetic fields the Boltzmann equation reads

$$
\frac{\partial f_{ \pm}}{\partial t} \pm Q\left(\mathbf{E}+\mathbf{v}_{\mathbf{k}} \times \mathbf{B}\right) \cdot \frac{\partial f_{ \pm}}{\partial \mathbf{k}}=\mathrm{I}_{ \pm}\left[f_{+}, f_{-}\right] .
$$

To linear order in the electric field we may parameterize the departure from equilibrium

$$
f_{ \pm}(\mathbf{k})=f_{0}\left(\varepsilon_{k}\right) \pm Q \mathbf{k} \cdot \mathbf{E} \psi(k)+\mathbf{k} .(\mathbf{E} \times \mathbf{B}) \psi_{\perp}(k),
$$

where for simplicity we focus on static solutions. Here, $\psi(k)$, represents the longitudinal shift due to the applied electric field, and $\psi_{\perp}(k)$ represents the transverse shift due to the combined electric and magnetic field. Substituting this expansion into (61) and retaining only terms up $\mathcal{O}(\mathbf{E})$

$$
\pm Q \mathbf{v}_{\mathbf{k}} \cdot\left[\mathbf{E}\left(\frac{\partial f_{0}}{\partial \varepsilon_{k}}\right) \mp Q(\mathbf{E} \times \mathbf{B}) \psi(k)+\mathbf{E}|\mathbf{B}|^{2} \psi_{\perp}(k)\right]=\mathrm{L}_{ \pm}[ \pm Q \mathbf{k} \cdot \mathbf{E} \psi]+\mathrm{L}_{ \pm}^{\prime}\left[\mathbf{k} .(\mathbf{E} \times \mathbf{B}) \psi_{\perp}\right]
$$

where the terms on the right hand side correspond to the distinct linearizations of the collision term arising from the longitudinal and transverse shifts of the distribution function — see Appendix A, These are straightforward, but rather tedious to derive, and involve momentum space integrals over the remaining products of Bose distribution functions. As noted by Damle and Sachdev, $\stackrel{20,21}{2}$ these integrals may be evaluated in terms of polylogarithm functions. Since the explicit form of these linearizations was not included in any of the original publications, $, 20,21$ we provide them in Appendix A. Although the details of the collision term are certainly important for a quantitative numerical implementation of the epsilon expansion, the physical results are primarily determined by the robust symmetry properties of these linearizations. In the case at hand, to lowest order in the epsilon expansion the collision terms may be neglected in accordance with our previous findings and we obtain

$$
\psi(k)=0, \quad \psi_{\perp}(k)=\frac{1}{|\mathbf{B}|^{2}}\left(-\frac{\partial f_{0}}{\partial \varepsilon_{k}}\right) .
$$

The vanishing of the longitudinal shift is consistent with the vanishing of the DC electrical conductivity, $\sigma_{x x}(B)$, in the drift regime. The heat current is readily found from the expansion (62)

$$
\mathbf{J}_{h}=2 \int \frac{d^{d} k}{(2 \pi \hbar)^{d}} \varepsilon_{k} \mathbf{v}_{k} \mathbf{k} \cdot(\mathbf{E} \times \mathbf{B}) \psi_{\perp}(k) .
$$


As such, to lowest order in the epsilon expansion

$$
\mathbf{J}_{h}=2 c^{2} \int \frac{d^{d} k}{(2 \pi \hbar)^{d}} \mathbf{k}\left(\mathbf{k} \cdot \mathbf{v}_{\mathrm{D}}\right)\left(-\frac{\partial f_{0}}{\partial \varepsilon_{k}}\right) .
$$

It is readily seen that this is consistent with our original transport coefficient (48). Indeed, our lowest order linear response solution (64), yields

$$
f_{ \pm}(\mathbf{k})=f_{0}\left(\varepsilon_{\mathbf{k}}\right)-\mathbf{k} \cdot \mathbf{v}_{\mathrm{D}}\left(\frac{\partial f_{0}}{\partial \varepsilon_{k}}\right)
$$

This coincides with the linearization of our exact boost distribution (54).

\section{Numerical Evaluation of $\alpha_{x y}$}

Having presented compelling evidence for the general form of the thermoelectric tensor, let us finally obtain the explicit numerical result within the epsilon expansion. Performing the angular integrals yields

$$
\alpha_{x y}=\frac{2 c^{2} S_{d}}{d B T(2 \pi \hbar)^{d}} \int_{0}^{\infty} d k k^{d+1} \frac{\beta e^{\beta \varepsilon_{\mathbf{k}}}}{\left(e^{\beta \varepsilon_{\mathbf{k}}}-1\right)^{2}}
$$

where $S_{d}=2 \pi^{d / 2} / \Gamma(d / 2)$ is the surface area of a unit hypersphere in $d$-dimensions. Introducing rescaled variables $\bar{k} \equiv c k / k_{B} T$ and $\tilde{m} \equiv m c^{2} / k_{B} T$ we find

$$
\alpha_{x y}=\frac{k_{B}}{B}\left(\frac{k_{B} T}{\hbar c}\right)^{d}\left[\frac{2 S_{d}}{d(2 \pi)^{d}} \int_{0}^{\infty} d \bar{k} \bar{k}^{d+1} \frac{e^{-\sqrt{\bar{k}^{2}+\tilde{m}^{2}}}}{\left(1-e^{-\sqrt{\bar{k}^{2}+\tilde{m}^{2}}}\right)^{2}}\right] .
$$

Within the epsilon expansion, $d=3-\epsilon$, and the mass parameter entering equation (69) is proportional to $\sqrt{\epsilon}$ as indicated in (14). To leading order we may thus evaluate the dimensionless numerical prefactor in three dimensions so as to obtain

$$
\alpha_{x y}=\frac{2 k_{B}}{B}\left(\frac{k_{B} T}{\hbar c}\right)^{3-\epsilon}\left[\frac{1}{6 \pi^{2}} \int_{0}^{\infty} d \bar{k} \frac{\bar{k}^{4} e^{-\bar{k}}}{\left(1-e^{-\bar{k}}\right)^{2}}\right]
$$

The term in square brackets is just our massless entropy prefactor, $\mathcal{C}_{3}=2 \pi^{2} / 45$, and we thus find

$$
\alpha_{x y}=\frac{4 \pi^{2}}{45} \frac{k_{B}}{B}\left(\frac{k_{B} T}{\hbar c}\right)^{3-\epsilon} .
$$

In particular, in $d=2$, one obtains

$$
\alpha_{x y}=\alpha_{0}\left(\frac{T^{2}}{B}\right) .
$$

where $\alpha_{0} \approx 0.88 k_{B}^{3}(\hbar c)^{-2}$. It is readily seen that both answers (71) and (72) are consistent with the general scaling arguments presented in section II Noting the inverse magnetic field dependence inherited from the drift velocity (31), it follows from equation (8) that the heat current scales as

$$
J_{h} \sim T^{2+(d-1) / z} \frac{E}{T^{1+1 / z}} \frac{T^{2 / z}}{B}=T\left(\frac{T^{d / z}}{B}\right) E=\tilde{\alpha} E
$$

in response to applied electric and magnetic fields. Our results are therefore consistent with the scaling behavior

$$
\alpha_{x y} \sim \frac{T^{d / z}}{B}
$$

when specialized to the Lorentz invariant case with $z=1$. This completes our initial survey of the drift regime in crossed electric and magnetic fields. 


\section{B. Collision Dominated Regime: $|\mathbf{E}|>c|\mathbf{B}|$}

Having discussed the drift regime in considerable detail, let us very briefly comment on our expectations when $E>c B$. As we discussed in section VIII, a single particle is continually accelerated by the electric field. Since the collisions in the QBE (12) conserve energy and momentum, there is no way to relax a transverse heat current, and so we expect $\alpha_{x y}$ to diverge at the clean fixed point in the absence of impurities. On the other hand, the regime $E>c B$ is continuously connected to that studied by Damle and Sachdev, $\stackrel{20,21}{\longleftarrow}$ with $E \neq 0$ and $B=0$. We thus expect the possibility of a finite electrical conductivity, $\sigma_{x x}(B)$. It would be interesting to check these expectations by including impurity scattering in the framework of the QBE, although we do not pursue this here.

\section{TEMPERATURE GRADIENT AND MAGNETIC FIELD}

Having discussed the heat current which flows in response to an electric field, we now turn our attention to the complementary problem of the electric current which flows in response to a temperature gradient. This is important in order to verify the Onsager reciprocity relations, $\frac{38,39,40}{2}$ which we have used at several points to recast $\tilde{\alpha}_{x y}=T \alpha_{x y}$. Although we no longer have the luxury of Lorentz invariance arguments, we will again argue in favor if two distinct regimes of behavior. We will begin in section IX A with a discussion of the drift regime. In section IX A 1 we will recover our previous results for $\alpha_{x y}$, by taking an appropriate moment of the QBE in a thermal gradient. In section IX A 2 we will further rederive this from a linearization of the collision term. Both of these approaches indicate the presence of a finite thermal conductivity, $\bar{\kappa}_{x x}(B)$, and we will examine this in more detail in section IX A3

\section{A. Drift Regime: $\nabla T \lesssim B$}

\section{Transverse electrical current in response to a temperature gradient}

Although we have obtained $\alpha_{x y}$ by several different routes in crossed electric and magnetic fields, it is prudent to consider the computation in a thermal gradient. The celebrated Onsager relations $\frac{38,39,40}{4 e l l}$ us that we ought to be able to compute $\alpha_{x y}$ by looking at the transverse electrical current which flows in response to a temperature gradient. Although this Onsager relation is expected to be true, it ought to be verified by direct calculation. An additional motivation to examine this complementary approach is that it will also pave the way to an analysis of the thermal conductivity, $\bar{\kappa}(B)$. Let us therefore drop the electric field from the Boltzmann equation (12) and introduce a temperature gradient. This is easily achieved by allowing the temperature variable to be a function of position $\underline{57}$ The generic form of the Boltzmann equation with $\mathbf{E}=0$ reads

$$
\frac{\partial f_{ \pm}}{\partial t}+\mathbf{v}_{\mathbf{k}} \cdot \frac{\partial f_{ \pm}}{\partial \mathbf{x}} \pm Q\left(\mathbf{v}_{\mathbf{k}} \times \mathbf{B}\right) \cdot \frac{\partial f_{ \pm}}{\partial \mathbf{k}}=\mathrm{I}_{ \pm}\left[f_{+}, f_{-}\right] .
$$

In the absence of any material inhomogeneity we may assume that any spatial variation is due to the imposed temperature gradient:

$$
\frac{\partial f_{ \pm}}{\partial \mathbf{x}}=\nabla_{\mathbf{x}} T\left(\frac{\partial f_{ \pm}}{\partial T}\right)
$$

Within linear response in $\nabla T$, we may replace $f_{ \pm}$by $f_{0}$ in the spatial gradient term. In this way we may write

$$
\frac{\partial f_{ \pm}}{\partial t}+\mathbf{v}_{\mathbf{k}} \cdot \nabla_{\mathbf{x}} T\left(-\frac{\varepsilon_{k}}{T} \frac{\partial f_{0}}{\partial \varepsilon_{k}}\right) \pm Q\left(\mathbf{v}_{\mathbf{k}} \times \mathbf{B}\right) \cdot \frac{\partial f_{ \pm}}{\partial \mathbf{k}}=\mathrm{I}_{ \pm}\left[f_{+}, f_{-}\right] .
$$

A characteristic feature of the (single particle) drift regime is that there is no net energy gain during each revolution; the energy gained as a particle is accelerated under the electric field is lost on the reverse journey $\underline{\underline{51}}$ This suggests that we ought to look at the time variation of the total energy current within Boltzmann theory. We may do so directly from equation (77) by multiplying both sides by $\varepsilon_{k} \mathbf{v}_{k}$ and integrating over all momenta. For relativistic particles this is aided by the fact that $\varepsilon_{k} \mathbf{v}_{k}=c^{2} \mathbf{k}$ is proportional to the momentum:

$$
\frac{\partial \mathbf{J}_{E}^{ \pm}}{\partial t}+c^{4} \int \frac{d^{d} k}{(2 \pi \hbar)^{d}} \mathbf{k}(\mathbf{k} \cdot \mathbf{U})\left(\frac{\partial f_{0}}{\partial \varepsilon_{k}}\right) \pm Q c^{2} \int \frac{d^{d} k}{(2 \pi \hbar)^{d}} \mathbf{k}\left(\mathbf{v}_{k} \times \mathbf{B}\right) \cdot \frac{\partial f_{ \pm}}{\partial \mathbf{k}}=0
$$


where we define $\mathbf{U} \equiv(-\nabla T) / T$, and $\mathbf{J}_{E}^{ \pm}$are the energy currents pertaining to particles and holes. In writing this equation we have used the fact that the integral involving the collision term vanishes; the momentum is an example of a so-called summational invariant 58 These quantities play an important rôle in the hydrodynamic limit. Integrating the third term by parts one obtains

$$
\frac{\partial \mathbf{J}_{E}^{ \pm}}{\partial t}+c^{4} \int \frac{d^{d} k}{(2 \pi \hbar)^{d}} \mathbf{k}(\mathbf{k} \cdot \mathbf{U})\left(\frac{\partial f_{0}}{\partial \varepsilon_{k}}\right) \mp Q c^{2} \int \frac{d^{d} k}{(2 \pi \hbar)^{d}}\left(\mathbf{v}_{k} \times \mathbf{B}\right) f_{ \pm}=0
$$

where we have used the fact that the terms involving derivatives of $\mathbf{v}_{k}$ vanish. Adding the particle and hole solutions yields

$$
\frac{\partial \mathbf{J}_{E}}{\partial t}+2 c^{4} \int \frac{d^{d} k}{(2 \pi \hbar)^{d}} \mathbf{k}(\mathbf{k} \cdot \mathbf{U})\left(\frac{\partial f_{0}}{\partial \varepsilon_{k}}\right)-c^{2} \mathbf{J}_{e} \times \mathbf{B}=0,
$$

where $\mathbf{J}_{E}$ and $\mathbf{J}_{e}$ are the total heat and electrical currents defined by equations (15) and (16). If we impose the time independence of $\mathbf{J}_{E}$ we obtain

$$
\mathbf{J}_{e} \times \mathbf{B}=2 c^{2} \int \frac{d^{d} k}{(2 \pi \hbar)^{d}} \mathbf{k}(\mathbf{k} \cdot \mathbf{U})\left(\frac{\partial f_{0}}{\partial \varepsilon_{k}}\right)
$$

In particular, if we apply a temperature gradient, $U_{y}$, in the $y$-direction and a magnetic field $B_{z} \equiv B$ in the $z$-direction, one obtains a transverse electrical current

$$
-J_{e}^{x} B=2 c^{2} \int \frac{d^{d} k}{(2 \pi \hbar)^{d}} k_{y}^{2}\left(\frac{\partial f_{0}}{\partial \varepsilon_{k}}\right) U_{y} .
$$

From the defining relations (17) we thus obtain

$$
\alpha_{x y}=\frac{2 c^{2}}{d B T} \int \frac{d^{d} k}{(2 \pi \hbar)^{d}} k^{2}\left(-\frac{\partial f_{0}}{\partial \varepsilon_{k}}\right) .
$$

This is in agreement with our drift answer (48) and our complementary calculation (60) based on Lorentz invariance arguments in crossed $\mathbf{E}$ and $\mathbf{B}$ fields. By taking appropriate moments of the Boltzmann equation ${ }^{59}$ we have obtained the thermoelectric tensor without recourse to an explicit solution. Moreover, we have recovered the correct Onsager symmetry relation. In addition, the vanishing of $\dot{\mathbf{J}}_{E}$ indicates that the thermal conductivity is finite in this regime. We shall examine this further in section IX A3. Before doing this we first need to perform a linear response analysis in $\nabla T$.

\section{Explicit Construction of Linear Response in $\nabla T$}

In the presence of a temperature gradient and a magnetic field

$$
\frac{\partial f_{ \pm}}{\partial t}+c^{2} \mathbf{k} \cdot \mathbf{U}\left(\frac{\partial f_{0}}{\partial \varepsilon_{k}}\right) \pm Q\left(\mathbf{v}_{k} \times \mathbf{B}\right) \cdot \frac{\partial f_{ \pm}}{\partial \mathbf{k}}=\mathrm{I}_{ \pm}\left[f_{+}, f_{-}\right]
$$

where $\mathbf{U} \equiv(-\nabla T) / T$. To linear order in the temperature gradient we parameterize

$$
f_{ \pm}(\mathbf{k})=f_{0}\left(\varepsilon_{k}\right)+\mathbf{k} \cdot \mathbf{U} \psi(k) \pm Q \mathbf{k} .(\mathbf{U} \times \mathbf{B}) \psi_{\perp}(k) .
$$

Note that $\psi(k)$ and $\psi_{\perp}(k)$ are distinct from those defined previously in equation (62). Substituting (85) into (84) and retaining only those terms of $\mathcal{O}(\mathbf{U})$

$$
c^{2} \mathbf{k} .\left[\mathbf{U}\left(\frac{\partial f_{0}}{\partial \varepsilon_{k}}\right) \mp \frac{Q}{\varepsilon_{k}}(\mathbf{U} \times \mathbf{B}) \psi(k)+\frac{Q^{2} \mathbf{U}|\mathbf{B}|^{2}}{\varepsilon_{k}} \psi_{\perp}(k)\right]=\mathrm{L}_{ \pm}^{\prime}[\mathbf{k} . \mathbf{U} \psi]+\mathrm{L}_{ \pm}\left[ \pm Q \mathbf{k} .(\mathbf{U} \times \mathbf{B}) \psi_{\perp}\right]
$$

where $\mathrm{L}$ and $\mathrm{L}^{\prime}$ are the distinct linearizations of the collision term discussed in Appendix $\mathrm{A}$. To lowest order in the epsilon expansion we may drop the collision terms to obtain

$$
\psi(k)=0, \quad \psi_{\perp}(k)=\frac{\varepsilon_{k}}{Q^{2}|\mathbf{B}|^{2}}\left(-\frac{\partial f_{0}}{\partial \varepsilon_{k}}\right)
$$


The electrical current obtained from (85) yields

$$
\mathbf{J}_{e}=2 Q^{2} \int \frac{d^{d} k}{(2 \pi \hbar)^{d}} \mathbf{v}_{k} \mathbf{k} \cdot(\mathbf{U} \times \mathbf{B}) \psi_{\perp}(k) .
$$

Substituting (87) into (88) we find

$$
\mathbf{J}_{e}=2 c^{2} \int \frac{d^{d} k}{(2 \pi \hbar)^{d}} \mathbf{k} \mathbf{k} \cdot\left(\frac{\mathbf{U} \times \mathbf{B}}{|\mathbf{B}|^{2}}\right)\left(-\frac{\partial f_{0}}{\partial \varepsilon_{k}}\right) \text {. }
$$

This has a structure that is close to that of equation (66), and once again this yields our previous expression for $\alpha_{x y}$. However, in addition we will be able to go beyond this result and examine the thermal conductivity, $\bar{\kappa}_{x x}(B)$.

\section{Thermal Conductivity}

Thus far, we have used the QBE in a thermal gradient and a magnetic field to verify our previous result for $\alpha_{x y}$, and the Onsager relation. As we have seen, this is encoded in the lowest order $\mathcal{O}\left(\epsilon^{0}\right)$ solution given in equation (87). However, self consistency of this non-trivial result for $\psi_{\perp}(k)$, in turn induces a non-trivial longitudinal shift (and a finite thermal conductivity) at the higher order of $\mathcal{O}\left(\epsilon^{2}\right)$. As indicated by equation (86), the $\mathcal{O}\left(\epsilon^{0}\right)$ solution for $\psi_{\perp}(k)$ yields a non-trivial $\psi(k)$ at $\mathcal{O}\left(\epsilon^{2}\right)$ :

$$
\mp Q \mathbf{v}_{k} \cdot(\mathbf{U} \times \mathbf{B}) \psi(k)=\mathrm{L}_{ \pm}\left[ \pm Q \mathbf{k} .(\mathbf{U} \times \mathbf{B}) \psi_{\perp}(k)\right] .
$$

The right hand side of this equation is structurally similar to the linearization encountered by Damle and Sachdev 20 in the context of (zero magnetic field) electrical transport. The only crucial difference is that the electric field has been replaced by $\mathbf{U} \times \mathbf{B}$, where we recall that $\mathbf{U} \equiv(-\nabla T) / T$. Using their notation, equation (90) may be written

$$
\mp \mathbf{v}_{k} \cdot \mathbf{C} \psi(k)= \pm \mathbf{k} . \mathbf{C}\left\{-\epsilon^{2}\left(\frac{c}{\hbar}\right) \int_{0}^{\infty} d k_{1}\left[\psi_{\perp}(k) \mathrm{F}_{1}\left(k, k_{1}\right)+\psi_{\perp}\left(k_{1}\right) \mathrm{F}_{2}\left(k, k_{1}\right)\right]\right\},
$$

where we denote the combination $Q(\mathbf{U} \times \mathbf{B})=\mathbf{C}$ - see equation (A9). Here, $\mathrm{F}_{1}\left(k, k_{1}\right)$ and $\mathrm{F}_{2}\left(k, k_{1}\right)$ are non-trivial kernels which we provide in equations (A16), A17) and (A18) of Appendix A. In writing equation (91) we have also restored a factor of $c / \hbar$ which stems from requirement that the collision term have dimensions of $s^{-1}$; the kernels are dimensionless and the prefactor combines with the measure of integration. That is to say,

$$
\psi(k)=\epsilon^{2}\left(\frac{\varepsilon_{k}}{\hbar c}\right) \int_{0}^{\infty} d k_{1}\left[\psi_{\perp}(k) \mathrm{F}_{1}\left(k, k_{1}\right)+\psi_{\perp}\left(k_{1}\right) \mathrm{F}_{2}\left(k, k_{1}\right)\right] .
$$

In view of the explicit $\epsilon^{2}$ dependence of equation (92) we should evaluate $\varepsilon_{k}$ and $\psi_{\perp}(k)$ in the massless limit. As follows from equation (87)

$$
\psi_{\perp}(k) \rightarrow \frac{1}{Q^{2} B^{2}} \frac{\bar{k} e^{\bar{k}}}{\left(e^{\bar{k}}-1\right)^{2}} ; \quad \bar{k} \equiv \frac{c k}{k_{B} T} .
$$

It is readily seen from Appendix $\mathrm{A}$, that the non-trivial kernels $\mathrm{F}_{1}$ and $\mathrm{F}_{2}$, are in fact functions of the dimensionless variables $\bar{k}$ and $\bar{k}_{i}$; note that we worked in units where $\hbar=c=1$ there. Following Damle and Sachdev, ${ }^{20}$ we denote

$$
\mathrm{F}_{1}\left(k, k_{1}\right) \equiv \Phi_{1}\left(\bar{k}, \bar{k}_{1}\right), \quad \mathrm{F}_{2}\left(k, k_{1}\right) \equiv \Phi_{2}\left(\bar{k}, \bar{k}_{1}\right) .
$$

The longitudinal displacement may thus be written

$$
\psi(k)=\frac{\epsilon^{2}}{\hbar}\left(\frac{k_{B} T}{Q B c}\right)^{2} G(\bar{k})
$$

where we introduce a universal scaling function of the dimensionless variable $\bar{k}$

$$
G(\bar{k}) \equiv \bar{k} \int_{0}^{\infty} d \bar{k}_{1}\left[\frac{\bar{k} e^{\bar{k}}}{\left(e^{\bar{k}}-1\right)^{2}} \Phi_{1}\left(\bar{k}, \bar{k}_{1}\right)+\Phi_{2}\left(\bar{k}, \bar{k}_{1}\right) \frac{\bar{k}_{1} e^{\bar{k}_{1}}}{\left(e^{\bar{k}_{1}}-1\right)^{2}}\right]
$$


We plot an appropriate moment of this distribution in Fig. 4. The heat current may be obtained from (95) by combining (85) with the usual relation (16)

$$
\mathbf{J}_{h}=2 \int \frac{d^{d} k}{(2 \pi \hbar)^{d}} \epsilon_{k} \mathbf{v}_{k}(\mathbf{k} . \mathbf{U}) \psi(k) .
$$

It follows from the definitions (17) that the corresponding thermal conductivity is given by

$$
\bar{\kappa}_{x x}=\frac{2 c^{2}}{d T} \int \frac{d^{d} k}{(2 \pi \hbar)^{d}} k^{2} \psi(k) .
$$

Performing the angular integrals gives

$$
\bar{\kappa}_{x x}=\frac{2 c^{2}}{d T} \frac{S_{d}}{(2 \pi \hbar)^{d}} \int_{0}^{\infty} d k k^{d+1} \psi(k)
$$

where $S_{d}=2 \pi^{d / 2} / \Gamma(d / 2)$ is the surface area of a unit hypersphere in $d$-dimensions. Substituting the result (95) into (99) and rescaling the momentum integral yields

$$
\bar{\kappa}_{x x}=\epsilon^{2} k_{B} c\left(\frac{\hbar}{Q B}\right)^{2}\left(\frac{k_{B} T}{\hbar c}\right)^{d+3}\left(\frac{2 S_{d}}{d(2 \pi)^{d}}\right) \int_{0}^{\infty} d \bar{k} \bar{k}^{d+1} G(\bar{k}) .
$$

Equivalently

$$
\bar{\kappa}_{x x}=g \epsilon^{2}\left(k_{B} c\right) l_{B}^{4} \lambda_{T}^{-(d+3)}
$$

where

$$
l_{B} \equiv \sqrt{\frac{\hbar}{Q B}}, \quad \lambda_{T} \equiv \frac{\hbar c}{k_{B} T}
$$

are the magnetic length, and a suitable thermal wavelength respectively. The numerical coefficient is given by

$$
g=\frac{2 S_{d}}{d(2 \pi)^{d}} \int_{0}^{\infty} d \bar{k} \bar{k}^{d+1} G(\bar{k})
$$

From equation (101) we see that $\bar{\kappa}$ has dimensions $J k^{-1} m^{-(d-2)} s^{-1}$. This is consistent with the defining relations (17). In addition, it is readily seen that our expressions for the thermal conductivity are in agreement with the scaling form (8) with dynamical exponent $z=1$. To lowest order in the epsilon expansion, the integral (103) should be performed in $d=3$ :

$$
g=\frac{1}{3 \pi^{2}} \int_{0}^{\infty} d \bar{k} \bar{k}^{4} G(\bar{k}) .
$$

This equation mirrors (3.33) of Damle and Sachdev ${ }^{20}$ The extra factor of momentum arises because we are considering heat transport as opposed to electrical transport. In Fig. 4 we plot the universal scaling function $\bar{k}^{4} G(\bar{k})$. We find by numerical integration that

$$
g \approx 5.55
$$

In particular, in $d=2$, we find

$$
\bar{\kappa}_{x x}=\bar{\kappa}_{0}\left(\frac{T^{5}}{B^{2}}\right),
$$

where $\bar{\kappa}_{0} \equiv g k_{B}^{6} /\left(4 e^{2} \hbar^{3} c^{4}\right)$. That is to say, in stark contrast to the case where $B=0$, the thermal conductivity does not diverge, but is finite. ${ }^{5}$ Moreover, the dependence on $\epsilon^{2}$ is inversely related to that of the universal DC electrical conductivity

$$
\sigma_{Q}=\frac{\mathcal{N}_{\sigma}}{\epsilon^{2}}\left(\frac{4 e^{2}}{h}\right),
$$




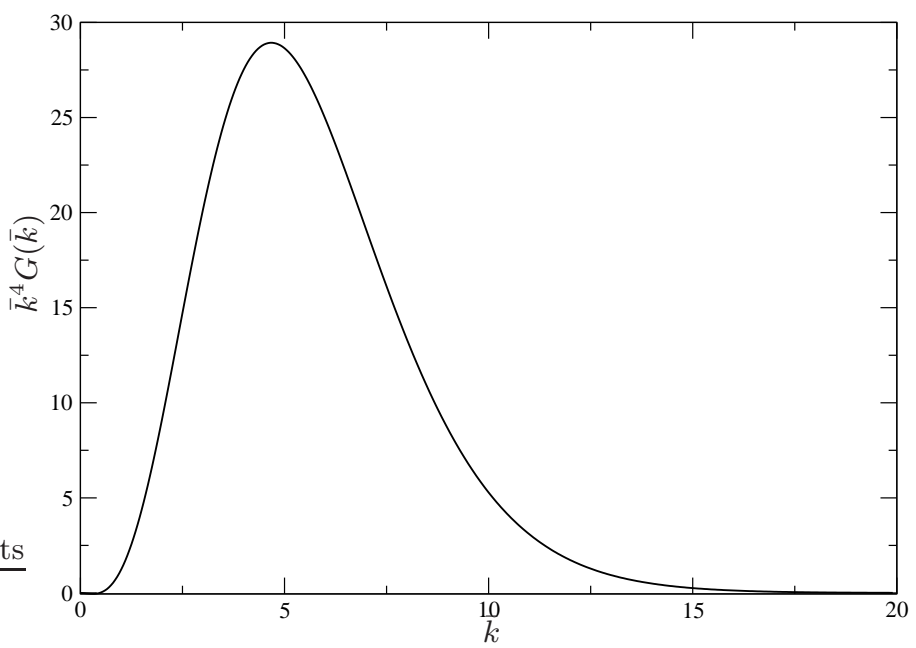

FIG. 4: Longitudinal scaling function, $G(\bar{k})$, required for computation of the thermal conductivity in the drift regime. This figure was obtained by truncating the upper limit of integration at $\bar{k}_{1}=100$.

where $\mathcal{N}_{\sigma} \approx 1.037$. When specialized to two-dimensions our result $\underline{\underline{5}}$ (101) may be cast in the equivalent form ${ }^{30}$

$$
\bar{\kappa}_{x x}=\mathcal{A}\left(\frac{\mathcal{S}_{\mathrm{ch}}^{2} T}{B^{2} \sigma_{Q}}\right)
$$

where, $\mathcal{S}_{\mathrm{ch}}=2 \mathcal{C}_{3} k_{B} \lambda_{\mathrm{T}}^{-2}$, is the entropy density of a charged scalar field calculated within the epsilon expansion, and the transformed dimensionless prefactor is given by

$$
\mathcal{A} \equiv \frac{g \mathcal{N}_{\sigma}}{8 \pi \mathcal{C}_{3}^{2}}
$$

With $g \approx 5.55, \mathcal{N}_{\sigma} \approx 1.037$, and $\mathcal{C}_{3}=2 \pi^{2} / 45$ one obtains $\mathcal{A} \approx 1.19$.

\section{HYDRODYNAMIC LIMIT AND INTERPOLATION}

The arguments presented above are manifestly iterative in the epsilon expansion and implicitly assume that $\epsilon^{2} \ll B$. It was subsequently pointed out in Ref. 30 using relativistic hydrodynamic arguments directly in $d=2$, that equation (108) holds as an exact relation in two dimensions with $\mathcal{A}=1$, or $g_{\mathrm{MHD}} \approx 4.66$. Moreover, it was shown that all the physical response functions are governed by combinations of thermodynamic variables and the single parameter $\sigma_{Q}$. In order to make contact with the hydrodynamic results of Ref. 30 we must study the ultra low field limit $B \ll \epsilon^{2}$. Even though $\epsilon^{2}$ is a small parameter, we must consider both the frequency and the field scales much smaller than this in order to enter the hydrodynamic regime. This is in conformity with the original zero field treatment of Damle and Sachdev $\underline{20}$ where the hydrodynamic crossover occurs at $\omega \sim \mathcal{O}\left(\epsilon^{2}\right)$. The magnetic field provides an additional cyclotron frequency and in the hydrodynamic limit this is assumed to be less than the scattering rate. As we shall discuss, the thermal conductivity in fact interpolates between these two closely separated limits $(\mathcal{A} \approx 1.19$ and $\mathcal{A}=1)$ as the ratio $B / \epsilon^{2}$ is varied. This mirrors recent findings in strictly two-dimensional graphene where (aside from the important aspects of spatial dimensionality and carrier statistics) the fine structure constant, $\alpha$, plays a similar rôle to $\epsilon \stackrel{34}{\underline{m}}$ In the subsequent discussion we derive the exact form of the hydrodynamic result analytically, using the QBE and the epsilon expansion around $d=3$. We will also discuss the interpolation as the magnetic field is varied.

Returning to our original equation (86), we wish to solve this coupled problem for the longitudinal and transverse distribution functions in more generality. It is convenient to decompose this relation in to two distinct equations which are respectively even and odd under reversal of the magnetic field:

$$
\mathbf{v}_{k} \cdot \mathbf{U}\left[\varepsilon_{k} \frac{\partial f_{0}}{\partial \varepsilon_{k}}+Q^{2} B^{2} \psi_{\perp}(k)\right]=\mathrm{L}_{ \pm}^{\prime}[\mathbf{k} \cdot \mathbf{U} \psi(k)]
$$

and

$$
\mp Q \mathbf{v}_{k} .(\mathbf{U} \times \mathbf{B}) \psi(k)=\mathrm{L}_{ \pm}\left[ \pm Q \mathbf{k} .(\mathbf{U} \times \mathbf{B}) \psi_{\perp}(k)\right]
$$


Direct elimination will yield equations governing the longitudinal and transverse response. In order to expose this more clearly, we use the expressions (A99) and (A26) for the distinct longitudinal and transverse linearizations of the collision term:

$$
\mathbf{v}_{k} \cdot \mathbf{U}\left[\varepsilon_{k} \frac{\partial f_{0}}{\partial \varepsilon_{k}}+Q^{2} B^{2} \psi_{\perp}(k)\right]=\mathbf{k} \cdot \mathbf{U}\left\{-\epsilon^{2}\left(\frac{c}{\hbar}\right) \int_{0}^{\infty} d k_{1}\left[\mathrm{~F}_{1}^{\prime}\left(k, k_{1}\right) \psi(k)+\mathrm{F}_{2}^{\prime}\left(k, k_{1}\right) \psi\left(k_{1}\right)\right]\right\},
$$

and

$$
\mp \mathbf{v}_{k} \cdot \mathbf{C} \psi(k)= \pm \mathbf{k} \cdot \mathbf{C}\left\{-\epsilon^{2}\left(\frac{c}{\hbar}\right) \int_{0}^{\infty} d k_{1}\left[\mathrm{~F}_{1}\left(k, k_{1}\right) \psi_{\perp}(k)+\mathrm{F}_{2}\left(k, k_{1}\right) \psi_{\perp}\left(k_{1}\right)\right]\right\}
$$

where $\mathbf{C} \equiv Q(\mathbf{U} \times \mathbf{B})$, and we have restored the factors of $\hbar$ and $c$ in the collision terms. The factors of k.U and k.C are readily cancelled leaving coupled integral equations for the distribution functions. For pedagogical purposes it is convenient to discretize these equations and write them in the simpler matrix form

$$
\begin{aligned}
-\varepsilon_{k} \frac{\partial f_{0}}{\partial \varepsilon_{k}}-Q^{2} B^{2} \psi_{\perp, k} & =\left(\frac{\epsilon^{2}}{\hbar c}\right) \varepsilon_{k} \mathrm{M}_{k, k_{1}}^{\prime} \psi_{k_{1}}, \\
\psi_{k} & =\left(\frac{\epsilon^{2}}{\hbar c}\right) \varepsilon_{k} \mathrm{M}_{k, k_{1}} \psi_{\perp, k_{1}},
\end{aligned}
$$

where we adopt the useful shorthand

$$
\begin{aligned}
\mathrm{M}_{k, k_{1}} \psi_{\perp, k_{1}} & \equiv \int_{0}^{\infty} d k_{1}\left[\mathrm{~F}_{1}\left(k, k_{1}\right) \psi_{\perp}(k)+\mathrm{F}_{2}\left(k, k_{1}\right) \psi_{\perp}\left(k_{1}\right)\right], \\
\mathrm{M}_{k, k_{1}}^{\prime} \psi_{k_{1}} & \equiv \int_{0}^{\infty} d k_{1}\left[\mathrm{~F}_{1}^{\prime}\left(k, k_{1}\right) \psi(k)+\mathrm{F}_{2}^{\prime}\left(k, k_{1}\right) \psi\left(k_{1}\right)\right] .
\end{aligned}
$$

Rearranging the first of equations (114) for $\psi_{\perp}$ and substituting in to the second immediately yields a Fredholm integral equation of the second kind for the longitudinal distribution function

$$
\psi_{k}=\psi_{k}^{\infty}-\left(\frac{\epsilon^{2}}{Q B \hbar c}\right)^{2} \varepsilon_{k} \mathrm{M}_{k, k_{1}} \varepsilon_{k_{1}} \mathrm{M}_{k_{1}, k_{2}}^{\prime} \psi_{k_{2}},
$$

where

$$
\psi_{k}^{\infty} \equiv\left(\frac{\epsilon^{2}}{Q^{2} B^{2} \hbar c}\right) \varepsilon_{k} \mathrm{M}_{k, k_{1}} \varepsilon_{k_{1}}\left(-\frac{\partial f_{0}}{\partial \varepsilon_{k_{1}}}\right)
$$

In the massless limit, we see that $\psi_{\infty}$ is nothing but our previous solution obtained from equations (92) and (93). It is recovered from the general integral equation (116) in the limit $B \gg \epsilon^{2}$. Employing dimensionless variables, $\bar{k} \equiv c k / k_{B} T$, and noting the rescaling of the integration measures in (115), the solutions of equation (116) are governed by the dimensionless prefactor of the second term which we may denote as

$$
r^{-1} \equiv \epsilon^{4}\left(\frac{l_{B}}{\lambda_{T}}\right)^{4}
$$

where $l_{B} \equiv \sqrt{\hbar / Q B}$ is the magnetic length, and $\lambda_{T} \equiv \hbar c / k_{B} T$ is the thermal wavelength. Equivalently,

$$
r \sim\left(\omega_{c}^{\text {typ }} \tau_{i n}\right)^{2}
$$

where $\tau_{i n}^{-1} \sim \epsilon^{2} k_{B} T / \hbar$ is the inelastic scattering rate due to the collisions at the clean fixed point, and $\omega_{c}^{\text {typ }} \sim$ $Q B /\left(k_{B} T / c^{2}\right)$ is the typical cyclotron rate of a thermal carrier $\stackrel{34}{2}$ In the limit, $r \rightarrow 0$, the particles experience a large number of collisions per typical period of revolution in the magnetic field. This corresponds to the hydrodynamic limit studied in Refs 30,34. In this respect, the magnetohydrodynamic parameter, $r$, plays a similar rôle to the Knudsen parameter in the development of Chapman-Enskog theory $\underline{\underline{58}}$ Deep in this hydrodynamic regime, $B \ll \epsilon^{2}$, the longitudinal distribution function satisfies the homogeneous equation

$$
\mathrm{M}_{k, k_{1}} \varepsilon_{k_{1}} \mathrm{M}_{k_{1}, k_{2}}^{\prime} \psi_{k_{2}}=0
$$


as follows directly from equation (116). In this limit, the distribution function projects on to a zero mode of the transverse operator $\mathrm{M}^{\prime}$. From our previous discussion of the transverse response (67) it is readily seen that

$$
f_{ \pm}(k)=f_{0}(k)+\mathcal{N} \mathbf{k} \cdot \mathbf{U}\left(-\frac{\partial f_{0}}{\partial \varepsilon_{k}}\right)+\text { transverse }
$$

is a zero mode of this collision operator. Moreover, this form would also emerge in a relaxation time approximation where $\mathcal{N} \equiv \tau_{\gamma} c^{2}$ and $\tau_{\gamma}$ is a suitable time scale. With the parameterization (85) we therefore consider

$$
\psi_{k}=\mathcal{N}\left(-\frac{\partial f_{0}}{\partial \varepsilon_{k}}\right)
$$

where $\mathcal{N}$ is a dimensionfull parameter to be determined. This normalization is fixed by the original inhomogeneous equation (116), and may be found by taking moments and extrapolating to the appropriate hydrodynamic limit. Placing the last term of equation (116) on the left hand side, and inverting the matrix operation in the definition of $\psi_{k}^{\infty}$, equation (116) may be rewritten in the equivalent form

$$
\left(\frac{Q^{2} B^{2} \hbar c}{\epsilon^{2}}\right)\left[\left(\mathrm{M}^{-1}\right)_{k, k_{1}} \varepsilon_{k_{1}}^{-1} \psi_{k_{1}}+\left(\frac{\epsilon^{2}}{Q B \hbar c}\right)^{2} \varepsilon_{k} \mathrm{M}_{k, k_{1}}^{\prime} \psi_{k_{1}}\right]=\varepsilon_{k}\left(-\frac{\partial f_{0}}{\partial \varepsilon_{k}}\right) .
$$

In order to make contact with the functional dependence (108) observed in the complementary regime, it is convenient to multiply this equation by $\varepsilon_{k}$ and integrate over all momenta. Upon sending $\psi_{k}$ to the hydrodynamic form (122), and working in the massless limit where $\varepsilon_{k}=c k$, one then obtains the normalization condition

$$
\mathcal{N} Q^{2} B^{2} \hbar c \int \frac{d^{d} k}{(2 \pi \hbar)^{d}} k \epsilon^{-2}\left(\mathrm{M}^{-1}\right)_{k, k_{1}} k_{1}^{-1}\left(-\frac{\partial f_{0}}{\partial \varepsilon_{k_{1}}}\right)=c^{2} \int \frac{d^{d} k}{(2 \pi \hbar)^{d}} k^{2}\left(-\frac{\partial f_{0}}{\partial \varepsilon_{k}}\right)
$$

where we exploit the fact that $\psi_{k}$ is a zero mode of $\mathrm{M}^{\prime}$. Using equation (48) we see that the right hand side of this equation is related to the entropy density. Moreover, using equation (3.28) of Damle and Sachdev, 20 we see that the left hand side involves the DC conductivity in the absence of a magnetic field. Recalling the main steps, we parameterize, $f_{ \pm}=f_{0} \pm Q \mathbf{k}$.E $\psi_{\mathrm{DS}}(k)$, and substitute into the QBE equation (61) with $\mathbf{B}=0$. To linear order in the electric field one obtains

$$
\frac{c}{k} \frac{\partial f_{0}}{\partial \varepsilon_{k}}=-\epsilon^{2}\left(\frac{c}{\hbar}\right) \mathrm{M}_{k, k_{1}} \psi_{\mathrm{DS}}\left(k_{1}\right)
$$

With this identification equation (124) may be recast in the form

$$
\mathcal{N} Q^{2} B^{2} c \int \frac{d^{d} k}{(2 \pi \hbar)^{d}} k \psi_{\mathrm{DS}}(k)=d T \frac{\mathcal{S}_{\mathrm{ch}}}{2},
$$

where $\mathcal{S}_{\mathrm{ch}}$ is the entropy density of a charged scalar field. This may be rearranged to read

$$
\mathcal{N} B^{2}\left[\frac{2 Q^{2} c}{d} \int \frac{d^{d} k}{(2 \pi \hbar)^{d}} k \psi_{\mathrm{DS}}(k)\right]=T \mathcal{S}_{\mathrm{ch}}
$$

Since the electric current is given by

$$
\mathbf{J}_{e}=2 Q^{2} \int \frac{d^{d} k}{(2 \pi \hbar)^{d}} \mathbf{v}_{k} \mathbf{k} \cdot \mathbf{E} \psi_{\mathrm{DS}}(k),
$$

the quantitity in square brackets is the conductivity. That is to say

$$
\mathcal{N}=\frac{T \mathcal{S}_{\mathrm{ch}}}{B^{2} \sigma_{Q}}
$$

where $\sigma_{Q}$ is the universal and non-trivial value of the DC electrical conductivity computed within the epsilon expansion in the absence of a magnetic field ${ }^{20}$ From this normalization, $\mathcal{N} \equiv \tau_{\gamma} c^{2}$, we may extract the characteristic damping

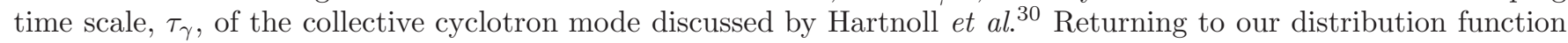
(122), the heat current is given by

$$
\mathbf{J}_{h}=2 \mathcal{N} \int \frac{d^{d} k}{(2 \pi \hbar)^{d}} \varepsilon_{k} \mathbf{v}_{k} \mathbf{k} \cdot \mathbf{U}\left(-\frac{\partial f_{0}}{\partial \varepsilon_{k}}\right)
$$




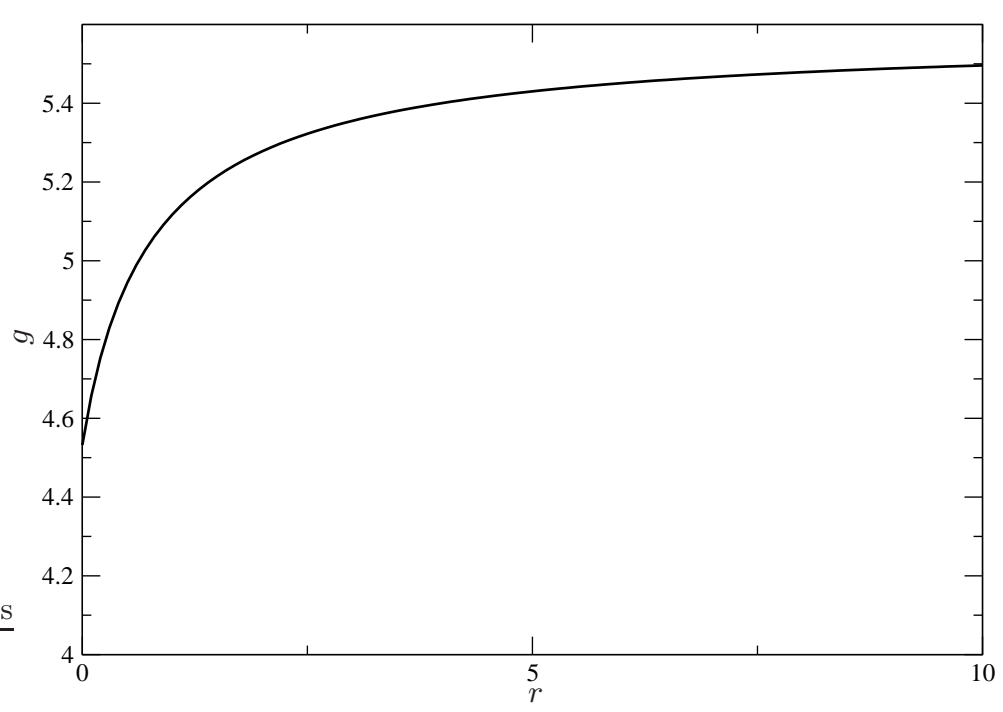

FIG. 5: Evolution of the dimensionless thermal conductivity prefactor, $g$, as the ratio, $r \propto B^{2} / \epsilon^{4}$, varies. Within the accuracy of our three dimensional Monte Carlo integrations (approximately 3\%) the result interpolates between the value reported in our previous Letter, ${ }^{\frac{5}{-}} g_{\infty} \approx 5.55$, and the magnetohydrodynamic value ${ }^{30} g_{0}=8 \pi\left(2 \pi^{2} / 45\right)^{2} /(1.037) \approx 4.66$. The latter corresponds to the exact relation, $\bar{\kappa}_{x x}(B)=T \mathcal{S}_{\mathrm{ch}}^{2} / B^{2} \sigma_{Q}$, for $B \ll \epsilon^{2}$.

The corresponding thermal conductivity reads

$$
\bar{\kappa}_{x x}=\frac{2 \mathcal{N} c^{2}}{d T} \int \frac{d^{d} k}{(2 \pi \hbar)^{d}} k^{2}\left(-\frac{\partial f_{0}}{\partial \varepsilon_{k}}\right) \equiv \mathcal{N} \mathcal{S}_{\mathrm{ch}},
$$

where we employ equation (48) again. Combining equations (129) and (131) one obtains the relation

$$
\bar{\kappa}_{x x}=\frac{T \mathcal{S}_{\mathrm{ch}}^{2}}{B^{2} \sigma_{Q}}
$$

deep in the hydrodynamic limit where $B \ll \epsilon^{2}$. We see that the functional dependence is the same in both limits, $B \gg \epsilon^{2}$, and $B \ll \epsilon^{2}$, and only the dimensionless prefactor is modified by a factor close to unity. As discussed in Appendix B numerical solution of the integral equation (116) yields the interpolation between these two regimes see Fig. 5, It is instructive to note that the hydrodynamic result (132), which holds exactly in two dimensions, is accessible within the framework of the epsilon expansion about $d=3$. The main delicate points are that we should work in a regime where $B \ll \epsilon^{2}$, and be careful to interpret the observables $\bar{\kappa}_{x x}, \sigma_{Q}$, and $\mathcal{S}_{\mathrm{ch}}$ by means of their respective epsilon expansions.

\section{CONCLUSIONS}

In this work we have examined the magnetothermoelectric response in the vicinity of a quantum critical point. We investigate the electrical and thermal transport and thermodynamics, and have presented general scaling arguments valid for arbitrary dimension, dynamical exponent and carrier statistics. These are supported by explicit calculations at the particle-hole symmetric SF-MI transitions of the Bose-Hubbard model. The presence of a magnetic field strongly influences the physical response, and we demonstrate the existence of a finite thermoelectric tensor, $\alpha_{x y}$, and a finite thermal conductivity, $\bar{\kappa}_{x x}(B)$, even in the absence of impurities. We relate these observations to a number of different approaches, based on Lorentz invariance, the quantum Boltzmann equation (QBE), and field theory considerations. In accordance with recent findings of Müller et al for graphene $\underline{\underline{34}}$ the dimensionless prefactor of our thermal conductivity is a smoothly varying function of $\omega_{c} \tau_{\text {in }}$. We derive an illuminating integral equation to describe this evolution, which exemplifies the rôle of zero modes in the hydrodynamic limit $\underline{\underline{34}}$ Our analytic and numerical calculations smoothly interpolate between the result presented in our previous Letter, $\frac{5}{,}$ and the limit of two-dimensional relativistic magnetohydrodynamics $\underline{\underline{30}}$ The recovery of the relativistic hydrodynamic relations within the framework of the epsilon expansion around three dimensions is quite compelling. Although the epsilon expansion is well established for the calculation of critical indices relating to thermodynamic quantities, it is much less widely 
employed in transport situations. The present body of results clearly demonstrate that we may address critical fluctuations in transport coefficients by using such methods. We see that the QBE approach not only has broad applicability but also provides a physically intuitive way to incorporate both quantum and thermal fluctuations in the hydrodynamic regime. Moreover, the results obtained are physically transparent and provide a platform for further studies.

\section{Acknowledgments}

We are extremely grateful to D. Basko, J.-S. Caux, J. Chalker, K. Damle, F. Essler, C. Hooley, D. Huse, A. Lamacraft, M. Müller, S. Sachdev, V. Oganesyan, B. Simons, and A. Tsvelik for helpful discussions at various stages of this work. This work was supported by NSF Grant No. DMR0213706, EPSRC Grants EP/E018130/1, EP/D050952/1, EP/D036194/1, and The Royal Society. We are particularly grateful to David Huse for insightful comments on the Lorentz transformations. MJB would also like to thank BNL and St. Andrews for hospitality during part of this work, the Rudolf Peierls Centre for Theoretical Physics, and the Cavendish Laboratory for financial support.

\section{APPENDIX A: COLLISION TERM AND POLYLOGARITHMS}

In order to make progress with the Boltzmann equation it is useful to linearize about an equilibrium Bose distribution. There are two distinct linearizations depending on the context. We gather some useful formulae below.

$$
\text { 1. } \delta f_{ \pm}(\mathbf{k})= \pm \mathbf{k} \cdot \mathbf{C} h(k)
$$

In this linearization we consider departures from equilibrium of the form $\delta f_{ \pm}(\mathbf{k})= \pm \mathbf{k}$. $\mathbf{C} h(k)$ where $\mathbf{C}$ is a constant vector, and $h(k)$ is a function of $|\mathbf{k}|$. This situation arises in the longitudinal response to an electric field,, $20 \mathbf{C}=Q \mathbf{E}$, and the transverse response to a temperature gradient, $\mathbf{C}=Q(\mathbf{U} \times \mathbf{B})$. We want to expand the collision term (13) to linear order in the applied field $\mathbf{C}$. We may write

$$
\mathrm{I}_{ \pm}=-\frac{2 u_{0}^{2}}{9} \int d \boldsymbol{\mu}\left(\mathcal{F}_{ \pm}^{\text {out }}-\mathcal{F}_{ \pm}^{\text {in }}\right)
$$

where, in units where $\hbar=c=1$,

$$
d \boldsymbol{\mu} \equiv \frac{1}{2 \varepsilon_{k}}\left[\prod_{i=1}^{3} \frac{d^{d} k_{i}}{(2 \pi)^{d}} \frac{1}{2 \varepsilon_{k_{i}}}\right](2 \pi)^{d} \delta\left(\mathbf{k}+\mathbf{k}_{1}-\mathbf{k}_{2}-\mathbf{k}_{3}\right)(2 \pi) \delta\left(\varepsilon+\varepsilon_{1}-\varepsilon_{2}-\varepsilon_{3}\right)
$$

represents the remaining phase space measure in equation (13). It is readily verified that

$$
\begin{aligned}
\mathcal{F}_{ \pm}^{\text {out }}-\mathcal{F}_{ \pm}^{\text {in }}= & \pm 3 \mathbf{k} \cdot \mathbf{C} h(k)\left(e^{\beta\left(\varepsilon_{2}+\varepsilon_{3}\right)}-e^{\beta \varepsilon_{1}}\right) n\left(\varepsilon_{1}\right) n\left(\varepsilon_{2}\right) n\left(\varepsilon_{3}\right) \\
& \pm \mathbf{k}_{1} \cdot \mathbf{C} h\left(k_{1}\right)\left(e^{\beta \varepsilon_{k}}-e^{\beta\left(\varepsilon_{2}+\varepsilon_{3}\right)}\right) n\left(\varepsilon_{k}\right) n\left(\varepsilon_{2}\right) n\left(\varepsilon_{3}\right) \\
& \pm 3 \mathbf{k}_{2} \cdot \mathbf{C} h\left(k_{2}\right)\left(e^{\beta \varepsilon_{3}}-e^{\beta\left(\varepsilon_{k}+\varepsilon_{1}\right)}\right) n\left(\varepsilon_{k}\right) n\left(\varepsilon_{1}\right) n\left(\varepsilon_{3}\right) \\
& \pm \mathbf{k}_{3} \cdot \mathbf{C} h\left(k_{3}\right)\left(e^{\beta\left(\varepsilon_{k}+\varepsilon_{1}\right)}-e^{\beta \varepsilon_{2}}\right) n\left(\varepsilon_{k}\right) n\left(\varepsilon_{1}\right) n\left(\varepsilon_{2}\right)+\mathcal{O}\left(\mathbf{C}^{2}\right)
\end{aligned}
$$

where $n(\varepsilon) \equiv f_{0}(\varepsilon)$ is the Bose distribution function, and we have used the identity $1+n(\varepsilon)=e^{\beta \varepsilon} n(\varepsilon)$. Upon substituting (A3) into (A1) it is convenient to interchange $\mathbf{k}_{3} \leftrightarrow \mathbf{k}_{2}$ in the last term. This transformation preserves the integration measure and the linearized collision term becomes

$$
\mathrm{I}_{ \pm} \rightarrow \mathrm{L}_{ \pm}= \pm\left(-\frac{2 u_{0}^{2}}{9}\right) \int d \boldsymbol{\mu}\left[\mathbf{k} \cdot \mathbf{C} h(k) \mathrm{T}_{1}\left(k_{1}, k_{2}, k_{3}\right)+\mathbf{k}_{1} \cdot \mathbf{C} h\left(k_{1}\right) \mathrm{T}_{2}\left(k, k_{2}, k_{3}\right)+\mathbf{k}_{2} \cdot \mathbf{C} h\left(k_{2}\right) \mathrm{T}_{3}\left(k, k_{1}, k_{3}\right)\right]
$$


where

$$
\begin{aligned}
\mathrm{T}_{1}\left(k_{1}, k_{2}, k_{3}\right) & \equiv 3\left(e^{\beta\left(\varepsilon_{2}+\varepsilon_{3}\right)}-e^{\beta \varepsilon_{1}}\right) n\left(\varepsilon_{1}\right) n\left(\varepsilon_{2}\right) n\left(\varepsilon_{3}\right), \\
\mathrm{T}_{2}\left(k, k_{2}, k_{3}\right) & \equiv\left(e^{\beta \varepsilon_{k}}-e^{\beta\left(\varepsilon_{2}+\varepsilon_{3}\right)}\right) n\left(\varepsilon_{k}\right) n\left(\varepsilon_{2}\right) n\left(\varepsilon_{3}\right), \\
\mathrm{T}_{3}\left(k, k_{1}, k_{3}\right) & \equiv 2\left(e^{\beta \varepsilon_{3}}-e^{\beta\left(\varepsilon_{k}+\varepsilon_{1}\right)}\right) n\left(\varepsilon_{k}\right) n\left(\varepsilon_{1}\right) n\left(\varepsilon_{3}\right) .
\end{aligned}
$$

As discussed by Damle and Sachdev $\underset{20}{2}$ to leading order in the epsilon expansion one may evaluate the necessary integrals directly in $d=3$. In addition, one may consider the massless limit where $\varepsilon_{k}=k$, and we have set $c=1$. The angular integrals and one of the radial integrals may be carried out explicitly, by means of formulas (C1), (C3) and (C5) of Ref. 20; in their notations $d^{3} k_{i} \equiv k_{i}^{2} d k_{i} d \Omega_{i}$. Interchanging $\mathbf{k}_{1} \leftrightarrow \mathbf{k}_{3}$ in the first term, and $\mathbf{k}_{1} \leftrightarrow \mathbf{k}_{2}$ in the last, this procedure yields

$$
\begin{aligned}
\mathrm{L}_{ \pm}= & \pm\left(-\frac{2 u_{0}^{2}}{9}\right) \frac{\mathbf{k} \cdot \mathbf{C}}{(4 \pi)^{3}} \int_{0}^{\infty} d k_{1} d k_{2}\left\{\frac{h(k)}{k^{2}} \mathrm{~T}_{1}\left(k_{1}+k_{2}-k, k_{2}, k_{1}\right) \mathrm{I}_{1}\left(k, k_{1}, k_{2}\right)\right. \\
& \left.-\frac{h\left(k_{1}\right)}{3 k^{4}}\left[\mathrm{~T}_{2}\left(k, k_{2}, k+k_{1}-k_{2}\right) \mathrm{I}_{2}\left(k, k_{1}, k_{2}\right)-\mathrm{T}_{3}\left(k, k_{2}, k+k_{2}-k_{1}\right) \mathrm{I}_{3}\left(k, k_{1}, k_{2}\right)\right]\right\},
\end{aligned}
$$

where $I_{1}, I_{2}$ and $I_{3}$ are (domain dependent) polynomials given in (C2), (C4) and (C6) of Ref. 20. In this way one may write the linearized collision term in the compact form ${ }^{20}$

$$
\mathrm{L}_{ \pm}= \pm \mathbf{k} \cdot \mathbf{C}\left\{-\epsilon^{2} \int_{0}^{\infty} d k_{1}\left[h(k) \mathrm{F}_{1}\left(k, k_{1}\right)+h\left(k_{1}\right) \mathrm{F}_{2}\left(k, k_{1}\right)\right]\right\}
$$

where we use the fact that $u_{0}=(24 / 5) \pi^{2} \epsilon$, and we define

$$
\mathrm{F}_{1}\left(k, k_{1}\right)=\frac{2 \pi}{25 k^{2}} \int_{0}^{\infty} d k_{2} \mathrm{~T}_{1}\left(k_{1}+k_{2}-k, k_{2}, k_{1}\right) \mathrm{I}_{1}\left(k, k_{1}, k_{2}\right)
$$

and

$$
\begin{array}{r}
\mathrm{F}_{2}\left(k, k_{1}\right)=-\frac{2 \pi}{75 k^{4}} \int_{0}^{\infty} d k_{2}\left[\mathrm{~T}_{2}\left(k, k_{2}, k+k_{1}-k_{2}\right) \mathrm{I}_{2}\left(k, k_{1}, k_{2}\right)\right. \\
\left.-\mathrm{T}_{3}\left(k, k_{2}, k+k_{2}-k_{1}\right) \mathrm{I}_{3}\left(k, k_{1}, k_{2}\right)\right] .
\end{array}
$$

We again emphasize that the functions $\mathrm{T}_{1}, \mathrm{~T}_{2}$ and $\mathrm{T}_{3}$ are evaluated in the massless limit. More explicitly

$$
\mathrm{F}_{1}\left(k, k_{1}\right)=\frac{6 \pi}{25} \frac{n\left(k_{1}\right)}{k^{2} n(k)} \int_{0}^{\infty} d k_{2} n\left(k_{2}\right)\left[1+n\left(k_{1}+k_{2}-k\right)\right] \mathrm{I}_{1}\left(k, k_{1}, k_{2}\right) .
$$

Likewise, if we denote

$$
\mathrm{F}_{2}\left(k, k_{1}\right) \equiv \mathrm{F}_{2}^{a}\left(k, k_{1}\right)+\mathrm{F}_{2}^{b}\left(k, k_{1}\right)
$$

then

$$
\begin{gathered}
\mathrm{F}_{2}^{a}\left(k, k_{1}\right)=\frac{2 \pi}{75} \frac{[1+n(k)]}{k^{4} n\left(k_{1}\right)} \int_{0}^{\infty} d k_{2} n\left(k_{2}\right) n\left(k+k_{1}-k_{2}\right) \mathrm{I}_{2}\left(k, k_{1}, k_{2}\right), \\
\mathrm{F}_{2}^{b}\left(k, k_{1}\right)=-\frac{4 \pi}{75} \frac{n(k)}{k^{4} n\left(k_{1}\right)} \int_{0}^{\infty} d k_{2} n\left(k_{2}\right)\left[1+n\left(k+k_{2}-k_{1}\right)\right] \mathrm{I}_{3}\left(k, k_{1}, k_{2}\right) .
\end{gathered}
$$

These expressions are in conformity with equations (C7) and (3.28) of Ref. 20. As noted by Damle and Sachdev, the integrals A12 , A14 and A15 may be evaluated exactly using polylogarithm functions. Although the method was carefully explained, the explicit form of these kernels was not stated in their original works. ${ }^{20,21}$ The expressions are quite lengthy, and are rather tedious to derive. Nonetheless, they are valuable for numerical work. We may write

$$
\mathrm{F}_{1}\left(k, k_{1}\right)=\frac{6 \pi}{25} \frac{n\left(k_{1}\right) n\left(k-k_{1}\right)}{k^{2} n(k)}\left[\Theta\left(k-k_{1}\right) \mu_{2}\left(k, k_{1}\right)-\Theta\left(k_{1}-k\right) \mu_{2}\left(k_{1}, k\right)\right],
$$


together with

$$
\mathrm{F}_{2}^{a}\left(k, k_{1}\right)=\frac{2 \pi}{75} \frac{[1+n(k)] n\left(k+k_{1}\right)}{k^{4} n\left(k_{1}\right)} \mathrm{L}_{2}^{a}\left(k, k_{1}\right)
$$

and

$$
\mathrm{F}_{2}^{b}\left(k, k_{1}\right)=\frac{4 \pi}{75} \frac{n(k) n\left(k_{1}-k\right)}{k^{4} n\left(k_{1}\right)}\left[\Theta\left(k-k_{1}\right) \mathrm{L}_{2}^{b}\left(k, k_{1}\right)-\Theta\left(k_{1}-k\right) \mathrm{L}_{2}^{b}\left(k_{1}, k\right)\right],
$$

where $\Theta\left(k-k_{1}\right)$ is the step function. In writing these kernels we have introduced

$$
\mathrm{L}_{2}^{a}\left(k, k_{1}\right)=24 \lambda_{4}^{-}\left(k, k_{1}\right)+12\left[k \eta_{3}\left(k, k_{1}\right)+k_{1} \eta_{3}\left(k_{1}, k\right)\right]-6 k k_{1} \lambda_{2}^{+}\left(k, k_{1}\right),
$$

where

$$
\begin{aligned}
& \lambda_{n}^{ \pm}(x, y) \equiv \beta^{-n}\left[\operatorname{Li}_{n}\left(e^{-\beta x}\right)+\operatorname{Li}_{n}\left(e^{-\beta y}\right) \pm \operatorname{Li}_{n}\left(e^{-\beta(x+y)}\right) \pm \operatorname{Li}_{n}(1)\right] \\
& \eta_{n}(x, y) \equiv \beta^{-n}\left[\operatorname{Li}_{n}\left(e^{-\beta x}\right)-\operatorname{Li}_{n}\left(e^{-\beta y}\right)-\operatorname{Li}_{n}\left(e^{-\beta(x+y)}\right)+\operatorname{Li}_{n}(1)\right]
\end{aligned}
$$

Further,

$$
\mathrm{L}_{2}^{b}\left(k, k_{1}\right)=-3\left[4 \mu_{4}+2\left(k-k_{1}\right) \mu_{3}-k k_{1} \mu_{2}+4 k_{1} \nu_{3}+2 k k_{1} \nu_{2}\right]
$$

where

$$
\mu_{n}(x, y) \equiv \beta^{-n}\left[\operatorname{Li}_{n}(1)+\operatorname{Li}_{n}\left(e^{-\beta x}\right)-\operatorname{Li}_{n}\left(e^{-\beta y}\right)-\operatorname{Li}_{n}\left(e^{-\beta(x-y)}\right)\right]
$$

and

$$
\nu_{n}(x, y) \equiv \beta^{-n}\left[\operatorname{Li}_{n}\left(e^{-\beta x}\right)-\operatorname{Li}_{n}\left(e^{-\beta y}\right)\right] .
$$

Here $\operatorname{Li}_{p}(z)$ is the polylogarithm with series expansion

$$
\operatorname{Li}_{p}(z)=\sum_{n=1}^{\infty} \frac{z^{n}}{n^{p}}
$$

Note that we have used the fact that $\mathrm{L}_{2}^{a}\left(k, k_{1}\right)$ is symmetric in order to eliminate the step functions from $\mathrm{F}_{2}^{a}\left(k, k_{1}\right)$. In writing these expressions we employ polylogarithms whose arguments lie within the unit disc. Although tedious to check analytically, the equality of these functions and the integral representations (A12), (A14) and (A15) is readily verified numerically. It is worth noting that the kernels $\mathrm{F}_{1}\left(k, k_{1}\right)$ and $\mathrm{F}_{2}\left(k, k_{1}\right)$ possess singularities when their arguments coincide.

$$
\text { 2. } \delta f_{ \pm}(\mathbf{k})=\mathbf{k} \cdot \mathbf{C} h(k)
$$

In this linearization we consider departures from equilibrium of the form $\delta f_{ \pm}(\mathbf{k})=\mathbf{k}$. C $h(k)$ where $\mathbf{C}$ is an arbitrary vector, and $h(k)$ is a function of $|\mathbf{k}|$. This charge independent situation arises in the longitudinal response to a temperature gradient, $\mathbf{C}=\mathbf{U} \equiv(-\nabla T) / T$, and in the transverse response to an electric field, $\mathbf{C}=\mathbf{E} \times \mathbf{B}$. In this case

$$
\begin{aligned}
\mathcal{F}_{ \pm}^{\text {out }}-\mathcal{F}_{ \pm}^{\text {in }} & =3 \mathbf{k} \cdot \mathbf{C} h(k)\left(e^{\beta\left(\varepsilon_{2}+\varepsilon_{3}\right)}-e^{\beta \varepsilon_{1}}\right) n\left(\varepsilon_{1}\right) n\left(\varepsilon_{2}\right) n\left(\varepsilon_{3}\right) \\
& +3 \mathbf{k}_{1} \cdot \mathbf{C} h\left(k_{1}\right)\left(e^{\beta\left(\varepsilon_{2}+\varepsilon_{3}\right)}-e^{\beta \varepsilon_{k}}\right) n\left(\varepsilon_{k}\right) n\left(\varepsilon_{2}\right) n\left(\varepsilon_{3}\right) \\
& +3 \mathbf{k}_{2} \cdot \mathbf{C} h\left(k_{2}\right)\left(e^{\beta \varepsilon_{3}}-e^{\beta\left(\varepsilon_{k}+\varepsilon_{1}\right)}\right) n\left(\varepsilon_{k}\right) n\left(\varepsilon_{1}\right) n\left(\varepsilon_{3}\right) \\
& +3 \mathbf{k}_{3} \cdot \mathbf{C} h\left(k_{3}\right)\left(e^{\beta \varepsilon_{2}}-e^{\beta\left(\varepsilon_{k}+\varepsilon_{1}\right)}\right) n\left(\varepsilon_{k}\right) n\left(\varepsilon_{1}\right) n\left(\varepsilon_{2}\right)+\mathcal{O}\left(\mathbf{C}^{2}\right) .
\end{aligned}
$$

Substituting this into the collision term, and again making the interchange $\mathbf{k}_{3} \leftrightarrow \mathbf{k}_{2}$ in the last term, we find

$$
\mathrm{I}_{ \pm} \leftrightarrow \mathrm{L}_{ \pm}^{\prime}=\left(-\frac{2 u_{0}^{2}}{9}\right) \int d \boldsymbol{\mu}\left[\mathbf{k} \cdot \mathbf{C} h(k) \mathrm{T}_{1}^{\prime}\left(k_{1}, k_{2}, k_{3}\right)+\mathbf{k}_{1} \cdot \mathbf{C} h\left(k_{1}\right) \mathrm{T}_{2}^{\prime}\left(k, k_{2}, k_{3}\right)+\mathbf{k}_{2} \cdot \mathbf{C} h\left(k_{2}\right) \mathrm{T}_{3}^{\prime}\left(k, k_{1}, k_{3}\right)\right]
$$


where

$$
\begin{aligned}
\mathrm{T}_{1}^{\prime}\left(k_{1}, k_{2}, k_{3}\right) & \equiv 3\left(e^{\beta\left(\varepsilon_{2}+\varepsilon_{3}\right)}-e^{\beta \varepsilon_{1}}\right) n\left(\varepsilon_{1}\right) n\left(\varepsilon_{2}\right) n\left(\varepsilon_{3}\right) \\
\mathrm{T}_{2}^{\prime}\left(k, k_{2}, k_{3}\right) & \equiv 3\left(e^{\beta\left(\varepsilon_{2}+\varepsilon_{3}\right)}-e^{\beta \varepsilon_{k}}\right) n\left(\varepsilon_{k}\right) n\left(\varepsilon_{2}\right) n\left(\varepsilon_{3}\right) \\
\mathrm{T}_{3}^{\prime}\left(k, k_{1}, k_{3}\right) & \equiv 6\left(e^{\beta \varepsilon_{3}}-e^{\beta\left(\varepsilon_{k}+\varepsilon_{1}\right)}\right) n\left(\varepsilon_{k}\right) n\left(\varepsilon_{1}\right) n\left(\varepsilon_{3}\right)
\end{aligned}
$$

In particular, it is readily seen that $T_{1}^{\prime}=T_{1}, T_{2}^{\prime}=-3 T_{2}$ and $T_{3}^{\prime}=3 T_{3}$. This procedure therefore yields

$$
\begin{aligned}
\mathrm{L}_{ \pm}^{\prime}= & \left(-\frac{2 u_{0}^{2}}{9}\right) \frac{\mathbf{k} \cdot \mathbf{C}}{(4 \pi)^{3}} \int_{0}^{\infty} d k_{1} d k_{2}\left\{\frac{h(k)}{k^{2}} \mathrm{~T}_{1}\left(k_{1}+k_{2}-k, k_{2}, k_{1}\right) \mathrm{I}_{1}\left(k, k_{1}, k_{2}\right)\right. \\
& \left.+\frac{h\left(k_{1}\right)}{k^{4}}\left[\mathrm{~T}_{2}\left(k, k_{2}, k+k_{1}-k_{2}\right) \mathrm{I}_{2}\left(k, k_{1}, k_{2}\right)+\mathrm{T}_{3}\left(k, k_{2}, k+k_{2}-k_{1}\right) \mathrm{I}_{3}\left(k, k_{1}, k_{2}\right)\right]\right\} .
\end{aligned}
$$

That is to say, the linearized collision terms may now be written

$$
\mathrm{L}_{ \pm}^{\prime}=\mathbf{k} . \mathbf{C}\left\{-\epsilon^{2} \int_{0}^{\infty} d k_{1}\left[\mathrm{~F}_{1}^{\prime}\left(k, k_{1}\right) h(k)+\mathrm{F}_{2}^{\prime}\left(k, k_{1}\right) h\left(k_{1}\right)\right]\right\}
$$

where

$$
\mathrm{F}_{1}^{\prime}\left(k, k_{1}\right) \equiv \mathrm{F}_{1}\left(k, k_{1}\right), \quad \mathrm{F}_{2}^{\prime}\left(k, k_{1}\right) \equiv 3\left(\mathrm{~F}_{2}^{b}\left(k, k_{1}\right)-\mathrm{F}_{2}^{a}\left(k, k_{1}\right)\right)
$$

and $\mathrm{F}_{1}\left(k, k_{1}\right), \mathrm{F}_{2}^{a}\left(k, k_{1}\right)$ and $\mathrm{F}_{2}^{b}\left(k, k_{1}\right)$ are given by equations (A16), (A17), and (A18) respectively.

\section{APPENDIX B: LONGITUDINAL CROSSOVER EQUATION}

As discussed in section $\mathrm{X}$, the thermal conductivity is a function of the dimensionless parameter, $r \propto B^{2} / \epsilon^{4}$, which controls the ratio of the typical cyclotron frequency for a thermal carrier to the inelastic scattering rate. The longitudinal distribution function satisfies the integral equation (116)

$$
\psi_{k}=\psi_{k}^{\infty}-\left(\frac{\epsilon^{2}}{Q B \hbar c}\right)^{2} \varepsilon_{k} \mathrm{M}_{k, k_{1}} \varepsilon_{k_{1}} \mathrm{M}_{k_{1}, k_{2}}^{\prime} \psi_{k_{2}}
$$

where $\psi_{k}^{\infty}, \mathrm{M}$ and $\mathrm{M}^{\prime}$ are given by equations (117) and (115) respectively. For both numerical and analytic purposes it is convenient to recast this equation in terms of the dimensionless momenta, $\bar{k} \equiv c k / k_{B} T$. This rescaling modifies the coefficient of $\psi_{k}^{\infty}$ given in equation (117), and it is convenient to introduce

$$
\psi_{k} \equiv \frac{\epsilon^{2}}{\hbar}\left(\frac{k_{B} T}{Q B c}\right)^{2} \Psi(\bar{k})
$$

and similarly for $\psi_{k}^{\infty}$. Adopting this rescaling the integral equation (116) may be recast in the dimensionless form

$$
\Psi(\bar{k})=\Psi_{\infty}(\bar{k})-r^{-1} \int_{0}^{\infty} d \bar{k}_{1}\left[\mathrm{Q}_{1}\left(\bar{k}, \bar{k}_{1}\right) \Psi(\bar{k})+\mathrm{Q}_{2}\left(\bar{k}, \bar{k}_{1}\right) \Psi\left(\bar{k}_{1}\right)\right]
$$

where

$$
r \equiv \frac{1}{\epsilon^{4}}\left(\frac{Q B}{\hbar}\right)^{2}\left(\frac{\hbar c}{k_{B} T}\right)^{4}
$$

and

$$
\Psi_{\infty}(\bar{k}) \equiv \bar{k} \int_{0}^{\infty} d \bar{k}_{1}\left[\frac{\bar{k} e^{\bar{k}}}{\left(e^{\bar{k}}-1\right)^{2}} \Phi_{1}\left(\bar{k}, \bar{k}_{1}\right)+\Phi_{2}\left(\bar{k}, \bar{k}_{1}\right) \frac{\bar{k}_{1} e^{\bar{k}_{1}}}{\left(e^{\bar{k}_{1}}-1\right)^{2}}\right]
$$


coincides with our previous distribution function (96). The non-trivial kernels, $\mathrm{Q}_{1}\left(\bar{k}, \bar{k}_{1}\right)$ and $\mathrm{Q}_{2}\left(\bar{k}, \bar{k}_{1}\right)$, are given by the integrals

$$
\mathrm{Q}_{1}\left(\bar{k}, \bar{k}_{1}\right) \equiv \int_{0}^{\infty} d \bar{k}_{2} \bar{k} \Phi_{1}\left(\bar{k}, \bar{k}_{1}\right) \bar{k} \Phi_{1}^{\prime}\left(\bar{k}, \bar{k}_{2}\right)
$$

and

$$
\mathrm{Q}_{2}\left(\bar{k}, \bar{k}_{1}\right) \equiv \int_{0}^{\infty} d \bar{k}_{2}\left[\bar{k} \Phi_{1}\left(\bar{k}, \bar{k}_{2}\right) \bar{k} \Phi_{2}^{\prime}\left(\bar{k}, \bar{k}_{1}\right)+\bar{k} \Phi_{2}\left(\bar{k}, \bar{k}_{1}\right) \bar{k}_{1} \Phi_{1}^{\prime}\left(\bar{k}_{1}, \bar{k}_{2}\right)+\bar{k} \Phi_{2}\left(\bar{k}, \bar{k}_{2}\right) \bar{k}_{2} \Phi_{2}^{\prime}\left(\bar{k}_{2}, \bar{k}_{1}\right)\right],
$$

where $\mathrm{F}_{1}\left(k, k_{1}\right) \equiv \Phi_{1}\left(\bar{k}, \bar{k}_{1}\right), \mathrm{F}_{2}\left(k, k_{1}\right) \equiv \Phi_{2}\left(\bar{k}, \bar{k}_{1}\right)$, and their primed counterparts, are the dimensionless kernels given in Appendix A Note that in deriving the results (B5), (B6) and (B7), we have also taken the massless limit in accordance with the epsilon expansion.

It is evident from the longitudinal crossover equation (B3), that the distribution function interpolates between $\Psi_{\infty}(\bar{k})$, as $r \rightarrow \infty$, and a zero mode of the integral operator in the hydrodynamic limit, $r \rightarrow 0$. In order to see this more formally it is natural to consider an expansion of the form

$$
\Psi(\bar{k})=\Psi_{0}(\bar{k})+r \Psi_{1}(\bar{k})+r^{2} \Psi_{2}(\bar{k})+\ldots
$$

Substituting this expansion into equation (B3) and equating coefficients at order $r^{-1}$ one finds that for a non-vanishing $\Psi_{0}(\bar{k})$ to be present it must be an exact zero mode of the integral operator:

$$
\int_{0}^{\infty} d \bar{k}_{1}\left[\mathrm{Q}_{1}\left(\bar{k}, \bar{k}_{1}\right) \Psi_{0}(\bar{k})+\mathrm{Q}_{2}\left(\bar{k}, \bar{k}_{1}\right) \Psi_{0}\left(\bar{k}_{1}\right)\right]=0 .
$$

As discussed in section $\mathrm{X}$, the function

$$
\Psi_{0}(\bar{k}) \propto \frac{\partial f_{0}}{\partial \varepsilon_{k}} \propto \frac{e^{\bar{k}}}{\left(e^{\bar{k}}-1\right)^{2}} \equiv \mathcal{R}_{0}(\bar{k})
$$

satisfies this homogeneous condition. Although it will not concern us here, equating coefficients at higher order in $r$ leads to a recursive hierarchy of integral equations for the functions $\Psi_{l}(\bar{k})$. These are similar in spirit (if not in details) to those encountered in the Knudsen expansion of the Boltzmann equation; see for example $\S 6.2$ of the book by Harris $\stackrel{58}{=}$ Equation (B9) is particularly important from a numerical perspective, since any regularization which lifts this zero mode property (e.g. through truncation of the integration limits or through rounding errors) may potentially yield a solution starting at order $r$, instead of order $r^{0}$, as evident from equation (B8); on dimensional grounds this would yield a thermal conductivity of the Wiedemann-Franz form. In order to recover the exact nonvanishing hydrodynamic limit (132), it is essential that this zero mode feature is properly implemented. To this end, let us parameterize our solutions to the integral equation in the form

$$
\Psi(\bar{k}) \equiv \mathcal{R}_{0}(\bar{k}) \Phi(\bar{k}),
$$

where $\Phi(\bar{k})$ is the solution to be determined. Assisted by the exponential decay of the prefactor, we convert the integral equation (B3) into an approximate matrix equation by expanding $\Phi(\bar{k})$ in a basis of $N_{b}$ basis functions:

$$
\Phi(\bar{k})=\sum_{n=0}^{N_{b}-1} c_{n} f_{n}(\bar{k}) .
$$

Substituting this decomposition into equation (B3), multiplying by $\bar{k}^{2} f_{m}(\bar{k})$ and integrating (where we incorporate an extra factor of $\bar{k}^{2}$ for convergence purposes) one obtains the matrix equation

$$
\mathrm{A}_{m n} c_{n}=\mathrm{B}_{m},
$$

where, $\mathrm{A}_{m n} \equiv \mathrm{A}_{m n}^{\mathrm{I}}+r^{-1} \mathrm{~A}_{m n}^{\mathrm{II}}$, with explicit matrix elements

$$
\mathrm{A}_{m n}^{\mathrm{I}} \equiv \int_{0}^{\infty} d \bar{k} \bar{k}^{2} \mathcal{R}_{0}(\bar{k}) f_{m}(\bar{k}) f_{n}(\bar{k})
$$

and

$$
\mathrm{A}_{m n}^{\mathrm{II}} \equiv \int_{0}^{\infty} d \bar{k} d \bar{k}_{1} d \bar{k}_{2} \bar{k}^{2} f_{m}(\bar{k})\left[\mathcal{R}_{0}(\bar{k}) f_{n}(\bar{k}) \mathrm{Q}_{1}^{(\mathrm{A})}\left(\bar{k}, \bar{k}_{1}, \bar{k}_{2}\right)+\mathrm{Q}_{2}^{(\mathrm{A})}\left(\bar{k}, \bar{k}_{1}, \bar{k}_{2}\right) \mathcal{R}_{0}\left(\bar{k}_{1}\right) f_{n}\left(\bar{k}_{1}\right)\right]
$$


where $\mathrm{Q}_{i}^{(\mathrm{A})}\left(\bar{k}, \bar{k}_{1}, \bar{k}_{2}\right)$ denote the integrands (or arguments) of the integral representations (B6) and (B7). In addition

$$
\mathrm{B}_{m} \equiv \int_{0}^{\infty} d \bar{k} d \bar{k}_{1} \bar{k}^{3} f_{m}(\bar{k})\left[\bar{k} \mathcal{R}_{0}(\bar{k}) \Phi_{1}\left(\bar{k}, \bar{k}_{1}\right)+\Phi_{2}\left(\bar{k}, \bar{k}_{1}\right) \bar{k}_{1} \mathcal{R}_{0}\left(\bar{k}_{1}\right)\right]
$$

The dimensionless thermal conductivity parameter is defined as

$$
g \equiv \frac{1}{3 \pi^{2}} \int_{0}^{\infty} d \bar{k} \bar{k}^{4} \Psi(\bar{k})
$$

and is thus approximated as

$$
g \simeq \frac{1}{3 \pi^{2}} \sum_{n=0}^{N_{b}-1} c_{n} \int_{0}^{\infty} d \bar{k} \bar{k}^{4} \mathcal{R}_{0}(\bar{k}) f_{n}(\bar{k}) .
$$

Evaluating the matrix elements numerically (whilst implementing the zero mode condition $\mathrm{A}_{m 0}^{\mathrm{II}}=0$ exactly) we may solve the linear system of equations for the coefficients $c_{n}$. Using a basis of $N_{b}=7$ monomials $\left(1, k, \ldots, k^{6}\right)$ we plot $g$ as a function of $r$ in Fig. 5. It is readily seen that within the numerical accuracy of our Monte Carlo integrations (approximately $3 \%$ ) this dimensionless coefficient interpolates between the result reported in our Letter, $\frac{5}{5}$ and the

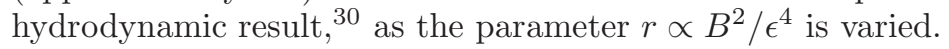

1 S. Sachdev, Quantum Phase Transitions (Cambridge University Press, 1999).

2 S. L. Sondhi, S. M. Girvin, J. P. Carini, and D. Shahar, Rev. Mod. Phys. 69, 315 (1997).

3 J. G. Bednorz and K. A. Müller, Z. Phys. B 64, 189 (1986).

4 M. Greiner, O. Mandel, T. Esslinger, T. W. Hänsch, and I. Bloch, Nature 415, 39 (2002).

${ }^{5}$ M. J. Bhaseen, A. G. Green, and S. L. Sondhi, Phys. Rev. Lett 98, 166801 (2007).

6 Z. A. Xu, N. P. Ong, Y. Wang, T. Kakeshita, and S. Uchida, Nature 406, 486 (2000).

7 Y. Wang, L. Li, M. J. Naughton, G. D. Gu, S. Uchida, and N. P. Ong, Phys. Rev. Lett. 95, 247002 (2005).

8 Y. Wang, L. Li, and N. P. Ong, Phys. Rev. B 73, 024510 (2006).

${ }^{9}$ I. Ussishkin, S. L. Sondhi, and D. A. Huse, Phys. Rev. Lett. 89, 287001 (2002).

10 I. Ussishkin, Phys. Rev. B 68, 024517 (2003).

11 V. Oganesyan and I. Ussishkin, Phys. Rev. B 70, 054503 (2004).

12 I. Ussishkin and S. Sondhi, Int. J. Mod. Phys. B 18, 3315 (2004).

13 S. Mukerjee and D. Huse, Phys. Rev. B 70, 014506 (2004).

14 V. Oganesyan, D. A. Huse, and S. L. Sondhi, Phys. Rev. B 73, 094503 (2006).

15 D. Podolsky, S. Raghu, and A. Vishwanath, Phys. Rev. Lett. 99, 117004 (2007).

${ }^{16}$ I. Ussishkin, D. A. Huse, and S. L. Sondhi, Critical scaling of thermal transport in model A dynamics for a superconductor, arXiv: cond-mat/0411040.

17 M. P. A. Fisher, P. B. Weichman, G. Grinstein, and D. S. Fisher, Phys. Rev. B 40, 546 (1989).

18 M. P. A. Fisher, G. Grinstein, and S. M. Girvin, Phys. Rev. Lett. 64, 587 (1990).

19 M.-C. Cha, M. P. A. Fisher, S. M. Girvin, M. Wallin, and A. P. Young, Phys. Rev. B 44, 6883 (1991).

${ }^{20}$ K. Damle and S. Sachdev, Phys. Rev. B 56, 8714 (1997).

21 K. Damle, Ph.D. thesis, Yale (1998).

22 A. G. Green and S. L. Sondhi, Phys. Rev. Lett. 95, 267001 (2005).

23 A. G. Green, J. E. Moore, S. L. Sondhi, and A. Vishwanath, Phys. Rev. Lett. 97, 227003 (2006).

24 D. S. Fisher, M. P. A. Fisher, and D. A. Huse, Phys. Rev. B 43, 130 (1991).

25 V. J. Emery and S. A. Kivelson, Nature 374, 434 (1995).

26 J. M. Kosterlitz and D. J. Thouless, J. Phys. C 6, 1181 (1973).

27 J. Šmakov and E. Sørensen, Phys. Rev. Lett. 95, 180603 (2005).

28 T. Senthil, unpublished.

29 M. Vojta, Y. Zhang, and S. Sachdev, Int. J. Mod. Phys. B 14, 3719 (2000).

30 S. A. Hartnoll, P. K. Kovtun, M. Müller, and S. Sachdev, Phys. Rev. B 76, 144502 (2007).

31 C. P. Herzog, P. Kovtun, S. Sachdev, and D. T. Son, Phys. Rev. D 75 (2007).

32 L. Fritz, J. Schmalian, M. Müller, and S. Sachdev, Phys. Rev. B 78, 085416 (2008).

33 M. Müller and S. Sachdev, Collective cyclotron motion of the relativistic plasma in graphene, arXiv:0801.2970.

${ }^{34}$ M. Müller, L. Fritz, and S. Sachdev, Quantum-critical relativistic magnetotransport in graphene, arXiv:0805.1413.

${ }^{35}$ R. Fazio and D. Zappalà, Phys. Rev. B 53, R8883 (1996).

36 S. R. deGroot, W. A. van Leeuwen, and C. G. van Weert, Relativistic Kinetic Theory (North-Holland, 1980).

37 J.-P. Blaizot, Nucl. Phys. B557, 183 (1999). 
38 A. A. Abrikosov, Fundamentals of the Theory of Metals (North-Holland, 1998).

39 L. Onsager, Phys. Rev. 37, 405 (1931).

40 L. Onsager, Phys. Rev. 38, 2265 (1931).

41 N. R. Cooper, B. I. Halperin, and I. M. Ruzin, Phys. Rev. B 55, 2344 (1997).

${ }^{42}$ M. P. A. Fisher, Phys. Rev. Lett. 65, 923 (1990).

43 X. G. Wen and A. Zee, Int. J. Mod. Phys. B4, 437 (1990).

44 E. Shimshoni, N. Andrei, and A. Rosch, Phys. Rev. B 68, 104401 (2003).

45 D. Jana, Nucl. Phys. B473, 659 (1996), erratum: Nucl. Phys. B485 (1997) 747-749.

46 D. Jana, Universal diamagnetism of charged scalar fields, cond-mat/9502103

47 W. Heisenberg and H. Euler, Z. Phys. 98, 714 (1936).

48 V. Weisskopf, Kong. Dans. Vid. Selsk. Math-fys. Medd. XIV No. 6 (1936), English translation in: Early Quantum Electrodynamics: A Source Book, A. I. Miller, Cambridge University Press, 1994.

49 J. Schwinger, Phys. Rev. 82, 664 (1951).

50 G. V. Dunne, in From Fields To Strings: Circumnavigating Theoretical Physics, edited by M. Shifman, A. Vainshtein, and J. Wheater (World Scientific, 2004), hep-th/0406216

51 J. D. Jackson, Classical Electrodynamics (John Wiley \& Sons, Inc., 1998), 3rd ed.

${ }^{52}$ V. Lukose, R. Shankar, and G. Baskaran, Phys. Rev. Lett 98, 116802 (2007).

53 J. I. Kapusta, Finite-temperature Field Theory (Cambridge University Press, 1989).

${ }^{54}$ W. Kohn, Phys. Rev. 123, 1242 (1961).

55 A. J. Schofield, private communication.

${ }^{56}$ Y. N. Obraztsov, Sov. Phys. (Solid State) 7, 455 (1965), [Fiz. Tver. Tela. $7573-581$ (1965)].

57 J. M. Ziman, Principles of the Theory of Solids (Cambridge University Press, 1964).

58 S. Harris, An Introduction to the Theory of the Boltzmann Equation (Dover Publications, 1971).

59 K. Huang, Statistical Mechanics (John Wiley and Sons, Inc., 1987), 2nd ed.

${ }^{60}$ We are grateful to Subir Sachdev for suggesting this clearer notation.

61 In general it is necessary to distinguish between the microscopic currents (15) and (16), and the transport currents (17), due to the presence of magnetization currents. ${ }^{41,56}$ This issue is circumvented in conventional Boltzmann approaches to transport in magnetic fields which do not include magnetization corrections to the distribution function.

62 Note that at weak fields, the $B^{2}$ contributions to the free energy are eliminated in these works by a renormalization of the electric charge associated with the Maxwell term.

${ }^{63}$ We are extremely grateful for illuminating conversations with David Huse on this point. 